\title{
Development of advanced inductive scenarios for ITER
}

T.C. Luce ${ }^{1}$, C.D. Challis ${ }^{2}$, S. Ide ${ }^{3}$, E. Joffrin ${ }^{4}$, Y. Kamada ${ }^{3}$, P.A. Politzer ${ }^{1}$, J. Schweinzer ${ }^{5}$, A.C.C. Sips $^{6}$, J. Stober ${ }^{5}$, G. Giruzzi ${ }^{4}$, C.E. Kessel ${ }^{7}$, M. Murakami ${ }^{8}$, Y.-S. Na ${ }^{9}$ J.M. Park ${ }^{8}$, A.R. Polevoi ${ }^{10}$, R.V. Budny ${ }^{7}$, J. Citrin ${ }^{11}$, J. Garcia ${ }^{4}$, N. Hayashi ${ }^{3}$, J. Hobirk ${ }^{5}$, B.F. Hudson ${ }^{12}$,

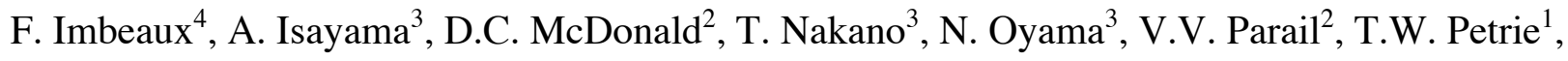
C.C. Petty ${ }^{1}$, T. Suzuki ${ }^{3}$, M.R. Wade ${ }^{1}$, the ITPA Integrated Operation Scenario topical group members and experts, the ASDEX-Upgrade team, the DIII-D team, JET EFDA contributors ${ }^{\mathrm{a}}$, and the JT-60U team

${ }^{1}$ General Atomics, PO Box 85608, San Diego, CA 92186-5608, USA

${ }^{2}$ Euratom/CCFE Fusion Association, Culham Science Centre, Abingdon, OX14 3DB, UK ${ }^{3}$ Japan Atomic Energy Agency, 801-1, Mukouyama, Naka, Ibaraki-ken 311-0193, Japan ${ }^{4}$ CEA, IRFM, 13108 Saint-Paul-lez-Durance, France

${ }^{5}$ Max-Planck-Institut für Plasmaphysik, Euratom Association, 85748 Garching, Germany ${ }^{6}$ JET-EFDA, Culham Science Centre, Abingdon, OX14 3DB, UK

${ }^{7}$ Princeton Plasma Physics Laboratory, PO Box 451, Princeton, NJ 08543-0451, USA

${ }^{8}$ Oak Ridge National Laboratory, PO Box 2008, Oak Ridge, TN 37831, USA

${ }^{9}$ Dept. of Nuclear Engineering, Seoul National U., 599 Gwanangno, Gwanak-Gu, Seoul, 151744 , Korea

${ }^{10}$ ITER Organization, Route de Vinon sur Verdon, 13115 St Paul Lez Durance, France

a See the Appendix of F. Romanelli et al., Proceedings of the 24th IAEA Fusion Energy Conference 2012, San Diego, US 
${ }^{11}$ FOM Institute DIFFER - Dutch Institute for Fundamental Energy Research, Association EURATOM-FOM, PO Box 1207, 3430 BE Nieuwegein, Netherlands

${ }^{12}$ University of California-San Diego, 9500 Gilman Dr., La Jolla, CA 92093-0417, USA

e-mail contact of main author: luce@fusion.gat.com

Abstract. Since its inception in 2002, the International Tokamak Physics Activity topical group on Integrated Operational Scenarios (IOS) has coordinated experimental and modeling activity on the development of advanced inductive scenarios for applications in the ITER tokamak. The physics basis and the prospects for applications in ITER have been advanced significantly during that time, especially with respect to experimental results. The principal findings of this research activity are as follows. Inductive scenarios capable of higher normalized pressure $\left(\beta_{\mathrm{N}} \geq 2.4\right)$ than the ITER baseline scenario $\left(\beta_{\mathrm{N}}=1.8\right)$ with normalized confinement at or above the standard H-mode scaling are well established under stationary conditions on the four largest diverted tokamaks (AUG, DIII-D, JET, JT-60U), demonstrated in a database of more than 500 plasmas from these tokamaks analyzed here. The parameter range where high performance is achieved is broad in $q_{95}$ and density normalized to the empirical density limit. MHD modes can play a key role in reaching stationary high performance, but also define the limits to achieved stability and confinement. Projection of performance in ITER from existing experiments uses empirical scalings and theory-based modeling. The status of the experimental validation of both approaches is summarized here. The database shows significant variation in the energy confinement normalized to standard H-mode confinement scalings, indicating the possible influence of additional physics variables absent from the scalings. Tests using the available information on rotation and the ratio of the electron and ion temperatures indicate neither of these variables in isolation can explain the variation in normalized confinement observed. 
Trends in the normalized confinement with the two dimensionless parameters that vary most from present-day experiments to ITER, gyroradius and collision frequency, are significant. Regression analysis on the multi-tokamak database has been performed, but it appears that the database is not conditioned sufficiently well to yield a new scaling for this type of plasma. Coordinated experiments on size scaling using the dimensionless parameter scaling approach find a weaker scaling with normalized gyroradius than the standard H-mode scaling. Preliminary studies on scaling with collision frequency show a favorable scaling stronger than the standard H-mode scaling. Coordinated modeling activity has resulted in successful benchmarking of modeling codes in the ITER regime. Validation of transport models using these codes on present-day expeirments is in progress, but no single model has been shown to capture the variations seen in the experiments. However, projection to ITER using these models is in general agreement with the favorable projections found with the empirical scalings.

PACs Nos: $\quad$ 52.55.Fa, 52.55.Dy, 52.25.Fi, 52.35.Py, 52.65.Tt 


\section{Introduction}

Operational scenarios with normalized fusion performance metrics significantly above those envisioned for the ITER baseline scenario (normalized pressure $\beta_{\mathrm{N}}=1.8$, confinement quality $\left.H_{98 \mathrm{y} 2}=1\right)$, but still relying on inductive current drive, should play a significant role in ITER. The ITER project has well-defined objectives [1]. There are two major physics objectives operation at fusion gain $Q=10$ with significant power and duration (500 MW for $>300 \mathrm{~s}$ ) and operation aiming for a true steady-state tokamak with $Q=5$. In addition to these objectives, maintaining the ability to reach higher fusion gain $(Q>20)$ is prescribed. Finally, the project is charged with testing technologies required for a fusion power plant, which means a significant fluence will be needed, albeit at a level well below that desired for certification for power plant use. With the exception of the steady-state objective, inductive scenarios with the advanced performance demonstrated on present-day tokamak experiments are attractive candidates for achieving each of these objectives for the ITER project.

To maximize the neutron fluence per plasma for the technology testing, it is necessary to extend the fusion burn duration [2]. This could be accomplished by reducing the plasma current, thereby saving transformer flux for flattop operation, and by using the auxiliary current drive systems to provide a significant fraction of the current to extend the duration. Of lesser significance but non-negligible is the increase in bootstrap current fraction that would be realized due to operation at lower current and higher pressure. The optimum current for this type of operation is expected to lie between that of the steady-state scenario and the baseline scenario, and it combines elements of both scenarios. Therefore, this mode of operation was called the ITER "hybrid" scenario, and the inductive scenarios discussed here are often referred to by this 
name. Beyond this potential to increase the fusion energy per pulse, the combination of higher normalized pressure and good confinement quality observed in present-day experiments opens other potential applications. For example, the pressure (and therefore the fusion power) at $q_{95}=4$ can be at or above that of the ITER baseline scenario. This opens the possibility of reaching the physics objective of energy gain $Q=10$ at $P_{\text {fus }}=500 \mathrm{MW}$ for $>300 \mathrm{~s}$ at reduced plasma current ( 11 MA) compared with that of the baseline scenario (15 MA). This would be particularly attractive because the risks associated with disruptions would be significantly reduced, due to the much lower energy stored in the poloidal magnetic field. The capacity for advanced performance in these scenarios would also be useful in the event that the confinement quality in the baseline scenario is less than anticipated. Operation at high-normalized pressure $\left(\beta_{\mathrm{N}}\right)$ may provide an alternative means to enhance the fusion power while still operating at $15 \mathrm{MA}$, rather than raising the current to $17 \mathrm{MA}$, which is the present contingency plan. Operation at high $\beta_{\mathrm{N}}$ and $15 \mathrm{MA}$ would also be a potential route to very high fusion gain $(Q>20)$ in the event the confinement quality is high. To take into account the broader possibilities of these scenarios for ITER, the term "advanced inductive" scenario, rather than the often-used term "hybrid" scenario, will be applied here to plasmas meeting the criteria of $\beta_{\mathrm{N}} \geq 2.4$ and $H_{98 \mathrm{y} 2} \geq 1$ in stationary operation $\left(t_{\mathrm{dur}} \geq 5 \tau_{\mathrm{E}}\right)$ in present-day experiments.

Several performance metrics will be used both for comparison among present-day tokamak results and projection to ITER. The normalized pressure $\beta_{\mathrm{N}} \equiv\langle p\rangle /\left\langle\mathrm{B}^{2}\right\rangle /(\mathrm{I} / \mathrm{aB})$ is the pressure normalized to a theoretical scaling that describes well the ideal MHD stability limit of a freeboundary plasma in the absence of a wall [3]. Here $p$ is the plasma pressure, B is the vacuum toroidal magnetic field at the geometric center of the plasma, I is the plasma current (in MA), and $a$ is the minor radius of the plasma (in MKS units except where noted). The ITER baseline 
scenario envisions operation at $\beta_{\mathrm{N}}=1.8$. To maintain the same fusion power at reduced current, $\beta_{\mathrm{N}}$ must increase inversely proportional to $\mathrm{I}$; hence, the threshold for advanced inductive operation was set to be $\beta_{\mathrm{N}}=2.4$. This is the level sufficient to maintain $500 \mathrm{MW}$ of fusion power at $75 \%$ of the current of the baseline scenario or $q_{95}=4$, where $q_{95}$ is the safety factor at the radius containing $95 \%$ of the total poloidal flux. The confinement quality $H_{98 y 2}$ is the thermal energy confinement time $\left(\tau_{\text {th }}\right)$ normalized to a scaling relation derived from a multitokamak database of edge localized moding (ELMing) H-mode plasmas [4]. A value of $H_{98 y 2}=1$ is used to estimate the performance of the ITER baseline scenario. For advanced inductive plasmas to be of use for the ITER physics and technical objectives, $H_{98 y 2}$ should be at least 1 , and this is used here as a threshold definition for advanced inductive operation. A figure of merit related to the energy gain, $G \equiv \beta_{\mathrm{N}} H_{89 \mathrm{P}} / q_{95}^{2}$ [2], is used to compare the potential fusion performance across tokamaks with different size and magnetic field. Here $H_{89 \mathrm{P}}$ is the ratio of the global energy confinement ( $\tau_{\mathrm{E}}$ ) to an L-mode scaling [5] and is used here to more easily relate confinement and stability. Confinement in $\mathrm{H}$ mode is about twice that in $\mathrm{L}$ mode, so $H_{89 \mathrm{P}}=2$ is an approximate figure of merit for good H-mode performance. The ITER baseline scenario performance with $Q=10$ has a value of $G=0.42$. The term "stationary" will be used to describe plasmas with inductive current drive in which the plasma parameters are not changing either on the energy confinement timescale or the global current relaxation timescale $\tau_{\mathrm{R}} \equiv 0.171 \mathrm{R} / \mathfrak{R}$, where $\mathrm{R}$ is the major radius of the plasma in meters and $\mathfrak{R}$ is the plasma resistance in $\mu \Omega$. The value of the resistance is not generally available, so $t_{\text {dur }} \geq 5 \tau_{\mathrm{E}}$ will be used as a practical criterion for the plasma being stationary, even though this is generally less than $\tau_{R}$. As a final operational metric, the ratio of the density to an empirical density limit 
$f_{\mathrm{DL}} \equiv n /\left(I / \pi a^{2}\right)[6]$ is used. A more complete discussion of these performance metrics can be found in [2].

The ITPA joint activities have focused on specific questions regarding advanced inductive scenarios. The earliest work focused on establishing the existence domain of the scenarios on the four largest divertor tokamaks (AUG [7], DIII-D [8], JET [9], JT-60U [10]) and looking for common ground in performance and phenomenology. This report will illustrate the commonality of the operational experience. A brief historical perspective is given in [2] and will not be repeated here. Having established a common ground, the joint activities turned to establishing a physics basis for applying these scenarios in ITER. Two key areas have been addressed - the stationary solution to the current profile evolution and projection of the energy confinement. The ITPA group has facilitated both joint experiments and modeling activities in these two areas, and these will be reported here. In addition to these two key questions, the challenge of operating at high performance while handling the steady state and transient heat flux to the divertor is beginning to be addressed. Initial experiments will be discussed. A summary of the prospects for applying these scenarios in ITER and the key remaining questions will be given.

\section{Existence domains}

Stationary plasmas meeting the criteria for an "advanced inductive" scenario have been demonstrated for $>3 \tau_{R}$ in the four largest divertor tokamaks (AUG, DIII-D, JET, JT-60U). The longest duration plasma, both in real time $(28 \mathrm{~s})$ and normalized to the resistive time $\left(>15 \tau_{R}\right)$, was achieved in JT-60U (figure 1) [10]. A database composed of parameters measured in 1129 plasmas from these four tokamaks has been compiled for use by the IOS topical group. Of these plasmas, 508 meet the criteria of $\beta_{\mathrm{N}} \geq 2.4$ and $H_{98 \mathrm{y} 2} \geq 1$ for defining advanced inductive 
operation for durations longer than $5 \tau_{\mathrm{E}}$. This duration criterion represents in all cases a significant fraction of $\tau_{\mathrm{R}}$. The data collected are a representative, but not exhaustive, sample of the experience in these tokamaks. A more complete description of the contents of the database is given in the appendix. Figure 2 shows the distribution of the data in current and density, using the dimensionless parameters $q_{95}$ and $f_{\mathrm{DL}}$ to facilitate comparison. The range of $q_{95}$ and $f_{\mathrm{DL}}$ shown represents the region of tokamak operating space of interest for fusion energy. Figure 2 clearly shows that advanced inductive operation has been obtained throughout this domain, indicating the robustness of the scenarios. Each of the tokamaks has achieved advanced inductive operation across a wide range of $q_{95}$. The DIII-D and JT-60U data lie primarily at $f_{\mathrm{DL}}<0.5$, while the JET data mostly lie at $f_{\mathrm{DL}}>0.6$. The AUG data span more uniformly the range of $f_{\mathrm{DL}}$, allowing connection to the other tokamaks. Another view of the operating space covered in this dataset is shown in figure 3 , where the achieved $\beta_{\mathrm{N}}$ in stationary discharges is plotted against the internal inductance $\ell_{\mathrm{i}}$. All four machines occupy roughly the same region of this parameter space. The JET $\ell_{i}$ data have been multiplied by a factor of $1 / 0.85$ because of the definition used. [JET reported $\ell_{\mathrm{i}}$ using a definition known as $\ell_{\mathrm{i}}(3)$, which is typically $15 \%$ lower than the usual definition of $\ell_{i}$, which is the ratio of the volume average of the squared magnitude of the poloidal magnetic field to the square of the average value of the poloidal magnetic field on the last closed flux surface. Alternate forms of $\ell_{i}$, such as $\ell_{i}(3)$ estimate the denominator using approximations to the boundary that permit analytic solutions, but which invalidate comparison of $\ell_{i}$ among plasmas of different shape.] All of these points lie within the specified range for the ITER poloidal field coils for $15 \mathrm{MA}$ operation $\left[0.6<\ell_{\mathrm{i}}(3)<1.2\right]$. It should be noted that the AUG dataset includes data from operations with both carbon and tungsten as the dominant plasma-facing material [11], and no data is supplied from operation in 
JET with the new beryllium-tungsten wall. No attempt has been made to distinguish the performance characteristics by wall material in any of the analysis presented here.

Figure 4 shows the achieved $\beta_{\mathrm{N}}$ versus proxies for the average value of the dimensionless gyroradius, collision frequency, and plasma current. Following reference 4, these are defined here as $\rho_{*} \propto\left(W_{\mathrm{th}} / n V\right)^{1 / 2} / B a, \quad v^{\dagger} \propto\left(n^{3} V^{2} / W_{\mathrm{th}}^{2}\right)\left(R^{5} / a^{3}\right)^{1 / 2}$, and $q_{\text {cyl }} \propto B V / I R^{2}$. [Later, proxies of the ratio of the thermal kinetic to magnetic pressure $\beta_{\mathrm{th}} \propto W_{\mathrm{th}} / V B^{2}$ and the ratio of the rotation speed to the ion sound wave phase velocity (Mach number) $M \propto \omega R\left(n V / W_{\text {th }}\right)^{1 / 2}$ will be used.] No definitive trend in the achieved $\beta_{\mathrm{N}}$ these dimensionless variables can be inferred from this dataset (figure 4); however, the higher $\beta_{\mathrm{N}}$ achieved at higher normalized collision frequency is consistent with a previously reported trend of $\beta_{\mathrm{N}}$ with higher density [12]. There is also an apparent correlation of $\beta_{\mathrm{N}}$ with low $\rho_{*}$ seen in figure 4(a). Such a correlation should appear if the achieved performance is set by the maximum power or magnetic field available in the tokamak. However, an examination of the individual points that form the edge of the dataset in that space for each tokamak does not support that mechanism as the cause of the boundary. It appears to be either an artifact of the sample provided or the parameter space explored by each tokamak.

The upper limit in $\beta_{\mathrm{N}}$ at which routine stationary operation can be obtained is set by the onset of $n=1$ tearing modes in each tokamak [8,13-15]. Because this limit is a resistive mode rather than an ideal mode, the result is often a dramatic loss of energy confinement rather than an immediate disruption. An unmitigated mode can slow the plasma rotation and continue to grow as a non-rotating mode, leading to disruption. However, the relatively slow growth of these 
modes allows detection and the potential for active means to recover the plasma performance or avoid a disruption.

At pressures lower than the maximum achieved, there appear to be systematic trends in the type of MHD observed $[9,10,13,16]$. Small sawteeth are generally seen for $q_{95}<4$, but are often absent at values of $q_{95}$ above this. The disappearance of sawteeth is often correlated with an increase in the stable operational $\beta_{\mathrm{N}}$ above which the $n=1$ tearing mode is seen. Given both the pressure and global confinement are generally higher than expected for conventional $\mathrm{H}$ mode, it is somewhat surprising that some type of MHD mode is observed in almost every plasma of this type. Fishbones are frequently observed in all four tokamaks, confirming that $\min (q)$ is near 1. In AUG and DIII-D, the fishbones tend to appear at higher density, while at lower density, $n>1$ tearing modes appear. It may be possible to make a map of the MHD behavior in operation space similar to figures 2 and 3 to quantify the commonality of the phenomena, but that is beyond the scope of this present work. The effects of MHD in the scenario will be discussed more fully in the next section.

\section{Projection to ITER}

Two key issues arise in projecting the plasma performance from present-day experiments to ITER. First, the scaling of confinement must be known. Only energy confinement has been studied so far - particle and momentum confinement are also important, but are not addressed here. Second, both the favorable confinement and stability properties of these scenarios may be tied to the current profile achieved under stationary conditions. The IOS group is working to address both issues with joint experimental and modeling activities. 


\subsection{Experiments}

Figure 5 shows that performance sufficient for $Q>5$ in ITER has been obtained across a variety of conditions, using the standard figure of merit $G$ described in section 1. For ITER, $G=0.3$ should be sufficient for $Q=5$, while $G=0.42$ should yield $Q=10$. As expected, $G$ increases significantly with increasing $\beta_{\text {th }}$ and decreasing $q_{\text {cyl }}$. Good performance has been

obtained across broad ranges of $\rho_{*}$ and $v^{\dagger}$. The key issue of this projection is whether the confinement scalings typically employed describe the variations seen in existing experiments. This point will be addressed in the remainder of this section.

Figure 6 shows how $H_{98 y 2}$ varies with the four dimensionless parameter proxies defined above for stationary plasmas with $\beta_{\mathrm{N}}>2.4$. (The criterion of $H_{98 y 2} \geq 1$ applied in the previous section on the existence domain has been relaxed.) For reference, the IPB98y,2 scaling in terms of dimensionless parameters is $\Omega_{\mathrm{i}} \tau_{\mathrm{th}, \mathrm{IPB} 98 \mathrm{y} 2} \propto \rho_{*}^{-2.69} \beta_{\mathrm{th}}^{-0.90} v^{\dagger, 0.08} q^{-3.0}$, where $\Omega_{\mathrm{i}}$ is the ion cyclotron frequency [17]. The first point to notice is that $H_{98 \mathrm{y} 2}$ varies by almost a factor of 2 across the database for each tokamak. This variation indicates that the reduced description of tokamak energy confinement given in the IPB98y,2 scaling developed for conventional H mode plasmas is either missing at least one variable that plays a significant role in the variation of energy confinement for the advanced inductive plasmas in this dataset or is not applicable at all to advanced inductive plasmas. It should be noted that variation of $H_{98 y 2}$ of a similar scale is seen in the data from which the scaling was derived when plotted against dimensionless parameter proxies [17]. On the applicability of $H_{98 y 2}$ to advanced inductive plasmas, it has been noted that the database from which the IPB98y,2 scaling was derived contains very little data with $\beta_{\mathrm{N}}>2.4$, which is defined here as the region of interest for advanced inductive plasmas 
(e.g., figure 7 of reference [18]). It is possible that extrapolation in this direction may not be valid. The topic of missing variables will be addressed later in this section. Some trends are apparent in these plots, despite the large variation at any given value of the dimensionless parameters. $H_{98 \mathrm{y} 2}$ increases with $\rho_{*}$, decreases with $\nu^{\dagger}$, and is roughly independent of $\beta_{\text {th }}$ and $q_{\text {cyl. }}$ There is a strong anti-correlation of $\rho_{*}$ and $v^{\dagger}$ in this dataset (figure 7), so it is not possible from this data alone to assign the variation to one or both of these variables. The origin of the correlation may be in the inverse temperature dependence of the gyroradius and the collision frequency or it may simply be an artifact of the experimental method or sample. A method to break this correlation is discussed later in this section. The fact that the ITER values of these two variables are significantly outside the existing dataset (both $>2 \mathrm{x}$ smaller in ITER) motivates understanding whether these trends reflect a true physical scaling, a consequence of the sample, or the failure of the $H_{98 \mathrm{y} 2}$ scaling to describe the true variation in energy confinement. The variation in $H_{98 \mathrm{y} 2}$ in this dataset is consistent with a scaling such as Bohm scaling $\left(B \tau_{\mathrm{E}} \propto \rho_{*}^{-2}\right)$ that has a weaker dependence on $\rho_{*}$ than that implicit in $H_{98 \mathrm{y} 2}$, which is closer to gyroBohm scaling $\left(B \tau_{\mathrm{E}} \propto \rho_{*}^{-3}\right)$. (See reference 17 for a general discussion of dimensionless parameter scaling and the definitions and implications of Bohm and gyroBohm scaling.) The trend for $H_{98 \mathrm{y} 2}$ to increase with reduced $v^{\dagger}$ is also consistent with improved confinement with $T_{\mathrm{i}}>T_{\mathrm{e}}$ and increased rotation associated with the unidirectional neutral beam heating that dominates this dataset. The JT-60U data makes rotation less likely as the cause of the variation, since those plasmas tend to have lower rotation from the more balanced neutral beam injection geometry in JT-60U. The trend is also consistent with the actual scaling being independent of $v^{\dagger}$ as seen in dedicated $v^{\dagger}$ experiments in H mode [17]; however, plots of the $H_{\mathrm{DS} 03}$ scaling [19] based on 
these H-mode dimensionless scaling experiments (figure 8) still show a clear decrease in confinement quality with $v^{\dagger}$. Note that the DS03 scaling appears to reduce the scatter slightly in the normalized confinement; this is particularly evident in the JT-60U data. Since JT-60U is the most different from the other tokamaks in its geometric parameters (aspect ratio, elongation), the DS03 scaling may describe these dependences better because of the method used to generate the scaling [19]. It is beyond the scope of this work to go beyond that speculation. The lack of variation of $H_{98 \mathrm{y} 2}$ with $\beta_{\mathrm{th}}$ may also be illusory, given that the DIII-D and JET data exhibit a trend if plotted for a specific range in $q_{95}$. It is important to recall that it is not the actual $\tau_{\mathrm{E}}$ that is being plotted here; therefore, the trends may reflect either dependencies in the data or weaknesses in the scaling used to normalize the data onto a common plot.

Dedicated experiments are needed to clarify the outstanding issues with the confinement projections; some of these have already been carried out. The dimensionless parameter scaling approach is well-suited to this task, since present-day experiments can operate at ITER values of $\beta$ and $q$ with reasonable approximations to the geometry of ITER. Then variations with $\rho_{*}, v^{\dagger}$, rotation, and $T_{\mathrm{i}} / T_{\mathrm{e}}$ can be made. Even if the ITER values are out of range of present experiments, by acting in concert, the experiments can corroborate results or extend the range of scans in order to yield projections with greater confidence. These experiments also provide critical data for validation of models used to project ITER performance.

To clarify the scaling with $\rho_{*}$, joint experiments between DIII-D and JET were carried out, including an identity match [20]. These experiments will be described in a separate publication, so only a brief indication of the results will be described here. Using DIII-D and JET in concert to extend the range in $\rho_{*}$ significantly beyond the possible range in either machine, it was possible to match profiles across a factor of 2.7 in $\rho_{*}$. The measured global scaling is close to 
Bohm scaling, and preliminary analysis of the local transport scaling is also closer to Bohm scaling than to the gyroBohm scaling typical of H-mode plasmas. This is consistent with the trend in $H_{98 \mathrm{y} 2}$ with $\rho_{*}$ seen in figures 6(a) and 8(a); i.e, the scalings have a stronger improvement in confinement with smaller $\rho_{*}$ than seen in the experiments. Note that direct extrapolation of these $\rho_{*}$ scaling experiments to ITER is not meaningful; the implied density in ITER would be much higher than the empirical density limit, because the collisionality in the present experiments is much higher than the ITER value. While the inferred $\rho_{*}$ scaling would imply a reduction in $H_{98 y 2}$ in the direction of ITER [as in figure 6(a)], this could be offset by a favorable scaling with $v^{\dagger}$ [consistent with the trend in figure 6(c)]. This points to the importance of knowing accurately both the gyroradius and collisionality scalings.

Dedicated joint experiments on the scaling with $v^{\dagger}$ have been proposed by the ITPA group. In an attempt to preview these experiments, the present database was examined to see if serendipitous scans of $v^{\dagger}$ could be found. The data was windowed in $\beta_{\mathrm{N}}(2.5-2.7)$ and the 0-D proxy for the $q_{\text {cyl }}(9.5-10.5)$ to locate candidate plasmas for matching. In this window, four plasmas from AUG, 93 from DIII-D, 17 from JET, and 2 from JT-60U appeared. For a perfect $v^{\dagger}$ scan with fixed poloidal cross section and aspect ratio, $I \propto B$, density is constant, and $T \propto B^{2}$ to keep $\rho_{*}, \beta$, and $q$ constant. This combination implies $v^{\dagger} \propto B^{-4}$. The AUG and JT$60 \mathrm{U}$ points did not have sufficient range in $B$, while the JET points did not hold the density constant across the range in $B$. In the DIII-D set, two suitable sets of five plasmas each were found. Table 1 gives the range in the essential variables. The resulting scaling from the two sets (figure 9) is approximately $B \tau_{\text {th }} \propto\left(v^{\dagger}\right)^{-0.5}$, which is at the upper end of the range of previous 
$v^{\dagger}$ scans in conventional $\mathrm{H}$ mode on DIII-D and JET [17]. (Here $\mathrm{B} \tau_{\text {th }}$ is the appropriate dimensionless proxy for thermal confinement time. See reference 17 for a more complete explanation.) This apparent scaling is consistent with the trends in figures 6(c) and 8(c) being true variations in confinement with $v^{\dagger}$, rather than artifacts of the data set. The facts that a $\rho_{*}$ scaling experiment cannot be carried out in present-day tokamaks at the ITER collisionality and that there is a significant reduction in $v^{\dagger}$ from present experiments to ITER, combined with these indications of a real dependence on $v^{\dagger}$ from the database, motivate the dedicated experiments proposed by the group.

It is clear from figures 6 and 8 that there is substantial variation in the normalized thermal confinement seen in the database at fixed values of any of the dimensionless parameters. One obvious explanation is that the variation arises from changes in parameters not accounted for in the standard engineering parameter scaling relations. The possible missing parameters that have been shown to influence confinement include the ratio of the ion to electron temperature $\left(T_{\mathrm{i}} / T_{\mathrm{e}}\right)$, $E \times B$ shearing of turbulence, and magnetic shear [17]. While no information on magnetic shear is available in the database, it is possible to test if either of the other two effects have a dominant influence on confinement by looking at data supplied in the original datasets. Unfortunately, these data are processed using different methods in each device, but they can be tested for the individual experiments to see if the temperature ratio or rotational shear alone is the dominant contributor to the variations in confinement seen here. This approach cannot rule out the possibility that combinations of these effects could play a significant role since a combined regression analysis is not possible.

Experiments with electron heating [ion cyclotron resonance heating (ICRH) and electron cyclotron heating $(\mathrm{ECH})$ on AUG [13] and ECH on DIII-D [21]) have varied the temperature 
ratio independent of the total energy and density. The AUG experiments show little variation in $H_{98 y 2}$ while the DIII-D experiments show some improvement of confinement when $T_{\mathrm{i}}>T_{\mathrm{e}}$, but the effect is weaker than that of rotation. It is necessary to take the change in rotation into account in these experiments, which compare confinement to co-injected neutral beam injection (NBI), because these electron-heating schemes add no torque, unlike the co-injected NBI. [The plasma response to this type of heating may include rotation, but it is not due to applied torque.] These results with electron heating are consistent with earlier observations of a weak dependence of confinement in density scans $[13,16]$, where $T_{\mathrm{i}} / T_{\mathrm{e}}$ approaches 1 as the density increases, due to collisional coupling. Quantifying these effects is important, since $\alpha$ heating will be dominantly electron heating and provide no torque input. From the data supplied here, it appears that the temperature ratio may play a role in the overall confinement quality, but cannot be the dominant factor in the variation of $H_{98 y 2}$ (figure 10). The temperature information supplied has been measured or processed differently in the four tokamaks, so it is not appropriate to display it in a single plot. (The JT-60U data are not shown in figure 10, since they have only a small variation in $T_{\mathrm{i}} / T_{\mathrm{e}}$ and $H_{98 \mathrm{y} 2}$ for plasmas where the $T_{\mathrm{i}}$ and $T_{\mathrm{e}}$ data are supplied.) The data from AUG is a simple average of point measurements in the range of normalized minor radius from $0.15-0.45$. The other tokamaks supplied a volume-averaged temperature for a portion of the dataset for both electrons and ions. The variations in the coverage of the measurements in radius, the fitting to magnetic coordinates, and the details of the volume averaging make a direct comparison among the machines difficult to justify. However, the datasets for AUG, DIII-D, and JET have sufficient variation in the temperature ratios (calculated self-consistently within the dataset) to draw some qualitative conclusions. For the AUG data [figure $10(\mathrm{a})]$, the normalized confinement $\left(H_{98 \mathrm{y} 2}\right)$ is quite good across the entire range of temperature ratios in the dataset, with the best confinement 
quality found for $T_{\mathrm{i}} / T_{\mathrm{e}}$ in the range 1.1-1.4. A simple linear regression only describes $4 \%$ of the variation in the data $\left(R^{2}=0.04\right)$. This indicates that the confinement quality is not correlated with the temperature ratio in these plasmas, nor is the temperature ratio the sole source of the variation seen in $H_{98 \mathrm{y} 2}$, consistent with previously reported results [22]. The DIII-D dataset does not access significantly the range of temperature ratios below 1 as does the AUG dataset, but the range in $H_{98 \mathrm{y} 2}$ seen at a temperature ratio near 1 is also seen at the higher ratios. A simple linear regression only describes $15 \%$ of the variation of $H_{98 \mathrm{y} 2}$, so the variation in $H_{98 \mathrm{y} 2}$ cannot be attributed solely to variation in the temperature ratio from the DIII-D data. This does not contradict the results in [21]; the hypothesis tested here is whether the variation in $T_{\mathrm{i}} / T_{\mathrm{e}}$ is a good predictor of the variation in $H_{98 y 2}$. The JET data provides a stronger case for the correlation of the overall confinement with temperature ratio $\left(R^{2}=0.538\right)$, so the temperature ratio may be responsible for a significant part of the variation in $H_{98 \mathrm{y} 2}$. Note however that at $T_{\mathrm{i}} / T_{\mathrm{e}}=1$, there is still a $\pm 20 \%$ variation in $H_{98 \mathrm{y} 2}$, so other effects are needed to fully describe the variation. Taken all together, the data provided here is consistent with the existence of a small effect of temperature ratio on confinement more clearly seen in experiments designed to isolate that effect. However, the variation in $H_{98 \mathrm{y} 2}$ seen across the entire dataset is unlikely to arise solely from the variation in the temperature ratio that was ignored in formulating that scaling relation.

Experiments on DIII-D that vary the applied torque at constant $\beta$ show a clear improvement in confinement with increasing rotation [23]. The value of $H_{98 \mathrm{y} 2}$ varies from near 1.0 to about 1.5 with roughly a linear trend for a factor of three increase in the Mach number or the absolute angular momentum, which are linearly correlated in the dataset. For the dataset compiled here, 
rotation data are available for only a fraction of the plasmas supplied by all four tokamaks (figure 11). Note that the rotation data is from different regions of the plasma in all four cases. The AUG data are a simple average of the measurements that lie in the range of normalized radius $\rho=0.15-0.45$, and the JET data come from near the magnetic axis, while the DIII-D and JT-60U are from $\rho=0.7-0.8$. The rotation is normalized to the global proxy defined previously for the ion sound speed to yield a coordinate proportional to the Mach number. The influence of rotation on confinement is attributed to shearing of the turbulent eddies due to a gradient in the flow in the plasma; however, a proxy of the Mach number could also be a proxy for the average shearing if the rotation data is from a fixed point in space and there is a no-slip boundary condition at the edge. Beginning with the AUG data [figure 11(a)], no obvious trend in $H_{98 \mathrm{y} 2}$ with Mach number is apparent, as indicated by $R^{2}=0.0003$ from a linear regression analysis. The DIII-D dataset has an irregular boundary and generally increases with Mach number [figure 11(b)] in a manner consistent with the data in [23], which is included here. But, as with the temperature ratio data, the linear regression explains only $15 \%$ of the variation of the data; therefore, the variation of the Mach number alone is not a good predictor of the variation in $H_{98 \mathrm{y} 2}$ in this dataset. The number of JET and JT-60U points is more limited, but the data from those tokamaks do not support an explanation of the variation in $H_{98 \mathrm{y} 2}$ as an effect solely due to rotation. It should be noted that the JT-60U dataset is unique in that it contains rotation data in both the co-current and counter-current directions. The edge rotation is strongly influenced by the radial electric field arising from particle losses due to the toroidal field ripple near the edge. This effect makes the proxy displayed here less likely to be a good proxy for rotational shearing. As with the temperature ratio, analysis of the data provided does not indicate that rotation (or its average gradient) is the sole source of the variation seen in $H_{98 \mathrm{y} 2}$ for the data provided. 
As noted previously, the $q$ profile or magnetic shear is also a candidate for explaining the variation in $H_{98 \mathrm{y} 2}$ in this dataset. Unfortunately, it is not obvious that there exists a good global proxy available to test this hypothesis. From the foregoing analysis, it appears that neither the variation of temperature ratio nor rotation presents a strong case as the sole source of the variation in $H_{98 \mathrm{y} 2}$. It is possible that the variation is due to a combination of effects not included in the basis for the $H_{98 \mathrm{y} 2}$ scaling or that the scaling simply does not describe the dependencies in this dataset.

An attempt has been made to do a multiple regression analysis on the existing dataset to yield a relation between $\tau_{\text {th }}$ and the standard engineering variables $(I, B, P, n, a, R, \kappa)$. Including all of these variables in a power law scaling yields $\tau_{\text {th }} \propto I^{0.79} B^{0.86} P^{-1.04} n^{0.36} R^{0.75} a^{1.67} \kappa^{-1.08}$. There are parameters from 579 plasmas in this regression. The scaling yields an excellent fit statistically $\left(R^{2}=0.96\right.$, rmse $\left.=8.6 \%\right)$, but it is unphysical to have such strong power degradation when many of the experiments were run using power feedback to maintain a specified level of stored energy. Reasonable fits can be made that have only three fit parameters $(I, P$, and either $a$ or $R$ ). The use of three parameters is somewhat justified by the observation that a principal components analysis of the fitting variables shows that $90 \%$ of the information content is in the first three eigenvalues. The two power law scalings found are $\tau_{\text {th }} \propto I^{1.41} P^{-0.67} R^{1.04}$ and $\tau_{\text {th }} \propto I^{1.21} P^{-0.48} a^{1.05}$. Comparison of the measured and fitted $\tau_{\mathrm{th}}$ is shown in figure 12 for both scalings. The statistical parameters of the fit with $R$ are somewhat better than for those from the fit with $a\left(R^{2}=0.89\right.$ vs 0.87 , rmse $=13.7 \%$ vs $15.0 \%$ for parameters from 613 plasmas), but $P$ has a stronger correlation with $R$ than with a (Table 2), leading to speculation that this correlation is the origin of the additional 
power degradation. This speculation is supported by the observation that the strong power degradation noted in the original seven-parameter fit disappears when $B$ is removed as a fitting variable. The correlation between $P$ and $a$ is not much less, and the overall fit is slightly poorer with $a$. It is clear from the figures that each of the tokamak datasets are not well-described by the scalings - the data tends to lie below the scaling at low confinement and rise above it at high confinement. The real conclusion of this discussion is that neither of these scalings should be considered as a conclusive predictor of thermal confinement time in advanced inductive plasmas. The uncanny similarity of the $P$ exponents to the IPB98y,2 and ITER-89P scalings leads to the speculation that similar correlations, perhaps arising from the operational boundaries of present-day tokamaks, might be found in the larger H-mode dataset.

Experiments have been carried out to broaden the physics basis for understanding the impact of the edge on advanced inductive scenarios and to apply standard steady state and transient heat flux control techniques to advanced inductive scenarios. AUG and DIII-D have carried out joint experiments on pedestal behavior [24]. The two main conclusions are that the pedestal continues to rise as more power flows out through the edge (contrary to some predictions) and that shape changes can have a significant impact on the pedestal parameters. Other than the stable access to higher $\beta_{N}$, there was no obvious distinction between the pedestal behavior in advanced inductive and standard H-mode plasmas. This indicates that the physics basis for pedestal behavior that has been developed over many years should also be applicable to advanced inductive plasmas. DIII-D has also extended the study of ELM mitigation using non-axisymmetric magnetic perturbations to advanced inductive scenarios [25]. Most of the same behavior observed in standard $\mathrm{H}$-mode plasmas using this method of ELM mitigation were also observed in the advanced inductive plasmas, e.g., density pumpout, little effect on the temperature pedestal, and 
the existence of a window in $q_{95}$ where the mitigation is effective. Confinement reduction similar to that seen in the larger conventional H-mode dataset is seen in advanced inductive plasmas with ELM mitigation. This again points to the utility for advanced inductive plasmas of the physics basis being formed on more conventional H-mode plasmas. Radiative divertor operation has been coupled with advanced inductive plasmas for steady-state heat flux reduction in AUG [26] and JET [27] using nitrogen and in DIII-D [28] using argon. The heat flux to the outer divertor strike point was reduced by a factor of 4 in JET and AUG and a factor of 2.5 in DIII-D. The JET plasmas reached $f_{\mathrm{DL}}=0.95$, but the edge plasma transitioned to Type III ELMs with an accompanying reduction in confinement quality to $H_{98 \mathrm{y} 2}=0.83$. The AUG plasmas only reached $f_{\mathrm{DL}}=0.8$, but saw improvement in the confinement quality over unseeded plasmas with $H_{98 y 2}$ reaching up to 1.3 . The confinement improvement was largely due to a surprising increase in pedestal temperature without any deleterious effects of impurity accumulation or radiation from the core. The DIII-D plasmas remained in the Type I regime with no reduction in confinement as the argon input was increased. Due to the "puff and pump" scheme used, the value of $f_{\mathrm{DL}}$ remained around 0.6. These initial results in applying steady state and transient heat flux mitigation techniques to advanced inductive plasmas are promising and should be explored with further experiments.

\subsection{Modeling}

Modeling activities in the IOS group have focused on the use of transport models to assess the performance in ITER. Only a brief summary of this work with references to existing publications is provided. In order to help establish a common basis for comparison, the ITPA group initiated a modeling activity to benchmark the heating and current drive sources (including $\alpha$ particles) and the transport model implementations in various codes in routine use throughout 
the world [29]. These codes have been compared on an ITER reference case under conditions expected for advanced inductive scenarios. The basic operating parameters $(B=5.3 \mathrm{~T}, I=$ 12 MA, reference plasma shape) were defined in the benchmark. The fuel and impurity density profiles were specified while the temperature, rotation, and current density profiles were calculated from self-consistent heating and current drive sources. For the external heating and current drive sources, the ITER reference designs at the time were used. After a few iterations within the group, the codes found very good agreement (about 10\% variation) in the global quantities such as $\alpha$ particle heating power, stored energy, and internal inductance. Agreement at the more detailed level of temperature profiles and power density of the heating was poorer but consistent with the level of agreement seen in the global quantities since they are integrals of the profiles. Nevertheless, the level of agreement found gave confidence that results of exploration studies of similar ITER scenarios should be free of basic implementation errors in the codes or version differences of the models. Subsequent unpublished work in the group improved upon the agreement shown in [29]. An example is shown in figure 13. Two benchmark tests have been performed using fixed density profiles. The first is evaluation of the electron and ion temperatures using the GLF23 transport model for fixed heating sources together with calculation of the self-consistent current density (inductive and noninductive). Four codes were involved in this benchmark: ASTRA [30], CRONOS [31], TOPICS [32] and TSC/TRANSP [33]. Figure 13(a) shows the temperatures computed with GLF23. Improved agreement with respect to previous analogous tests [29] is due to identification and reconciliation of differences in the implementation of GLF23 and in the NBI heating and current drive modules in the various codes. The second benchmark test was evaluation of the components of the current density for fixed temperatures. Figure 13(b) shows the total and bootstrap current densities for this test and figure 13(c) show the resulting safety factor profiles. The source of some of the remaining differences has been identified as arising from the methods the codes use to describe the 
magnetic equilibrium. The benchmark results have been archived by the group and are available by request to compare results to a standard case to modelers using codes not included in the original benchmark.

Simulations with models based on drift-wave theory or 0-D scalings have been compared to advanced inductive plasmas in existing experiments using measured profiles. Linear gyrokinetic stability analysis of plasmas from AUG, DIII-D, and JET found them to be remarkably stable, in some cases out to $\rho=0.7$ [34]. Given this, it is not too surprising that drift-wave models such as GLF23 and the Weiland model had difficulty reproducing the electron and ion temperature profiles in these plasmas. The ion temperature variation in DIII-D plasmas with an intentional reduction in the applied torque shows the variation in the ion temperature is described well by the GLF23 model only when the effects of $E \times B$ shearing are included in the calculation [23]. The electron temperature profiles are less sensitive to the rotation, which is reproduced by the GLF23 results. The electron temperature in DIII-D is uniformly under-predicted by GLF23 in these cases. The ion temperature variation in AUG plasmas with an intentional increase in heating power and torque is described well by the Weiland model [35]. This model over predicts the electron temperature in these cases. The resulting electron and ion temperature profiles calculated from Weiland model in these conditions are insensitive to $E \times B$ shearing when it is included in the calculation. None of these models has been conclusively shown to reproduce well the whole range of present-day experiments; therefore, modeling should be viewed as playing a role in suggesting further experiments and providing and indication of the sensitivity of the ITER projections to the various necessary assumptions, rather than a definitive prediction of ITER performance. 
With that caveat, a brief overview of the projections of advanced inductive performance to ITER is given here. It is difficult to characterize all of these projections, due to the large variety of assumptions that can and must be made to bridge the gap between present-day experiments and ITER. Two general types of projections have been made - those using experimental profiles plus scaling relations and those using combinations of profiles simulated with transport models with experimental or assumed profiles. Following the methodology detailed in [8], DIII-D plasmas were projected to ITER scenarios at $\sim 10$ and $14 \mathrm{MA}$ using three different confinement scalings $[8,12]$. At the lower current, the projected gain runs between $3.0-9.0$ for the more pessimistic ITER89-P scaling, between 4.7-8.9 for the IPB98y,2 scaling, and >20 for the more optimistic DS03 scaling based on dimensionless parameter scaling experiments. At the higher current, $Q>10$ is projected for ITER-89P and $>30$ for the H-mode scalings. A similar method was used to project AUG plasmas to ITER at 10,12 , and 14 MA [22]. The projected gains are $6.5,>10$, and $>30$, respectively. A mixed model and scaling approach was used to project JET plasmas to $\sim 11$ and 14 MA in ITER [9]. The radial profiles were generated by a selfconsistent transport calculation, but the overall confinement was normalized to agree with either the IPB98y, 2 scaling or a gyroBohm scaling. The projected gain at the lower current ranges from 2.2-4.6 and from 5.4-7.8 at the higher current. Increasing the density peaking is seen to be beneficial for increased gain, given the assumptions in this modeling. Similar methods were used combining the GLF23 transport model to give the radial profiles of temperature [34] for 13 MA plasmas in ITER. In some cases, the transport model was rescaled to match a specific scaling projection. The projected gains range from $Q=4-13$, with density peaking and pedestal height identified as critical parameters in the projections. Projections using transport models for the temperature profiles (fixed density and impurity profiles with no rotation) tested the sensitivity 
of the projections to the mixture of heating and current drive sources [29]. These will affect the resulting current profile, which is a parameter in the transport models. The variations reported for 12 MA ITER plasmas are $Q=5.6-6.8$. It should be noted that the current evolution leads to $q<1$ in the plasma center in those simulations, which is not typically observed in present-day experiments. This point will be discussed further below.

A few generalizations about the sensitivity of the ITER projections to the assumptions in the simulations can be drawn. A key conclusion is that a variety of methods and assumptions indicate the gain realized in ITER with advanced inductive scenarios would be sufficient to accomplish many of the project objectives. To increase confidence in these projections, it will be necessary to justify the most critical assumptions or replace them with validated models. A few of these critical assumptions, as highlighted by the modeling activities will be discussed here.

As with projections of the baseline scenario to ITER, the assumption about the pedestal height is critical to the performance projection. Recent studies have shown that the GLF23 model is sensitive to the shape of the $q$ profile and suggest that the volume average of $s / q$ up to the pedestal top may be a key parameter for energy confinement [36]. (Here $s$ is the magnetic shear.) This points to the need to understand the mechanisms by which the current profile comes into equilibrium in these plasmas. The peaking of the density profile has a clear influence on the projected fusion performance. Modeling of the fuel density profile is rare, while modeling of the behavior of the helium ash is usually done on a 0-D basis. Modeling of the core transport of the impurities envisioned for heat flux mitigation has not been carried out for advanced inductive plasmas and could be a weak point for any improved confinement regime.

A method to map the allowed operational space including the constraints of the density limit, maintaining $\mathrm{H}$ mode, and flux consumption has been developed [37]. Figure 14 shows such a 
diagram for long-pulse advanced inductive operation. This diagram is generated by evaluating 0-D formulas for the scenario limits mentioned above while choosing as constraints other physics or operational goals. The operational space is then displayed in terms of any unspecified parameters. Specifically, the analysis shown in figure 14 begins with setting the desired values of three parameters - the plasma duration, the input power, and the fusion gain. From these, one can use the design values of ITER to derive the density and temperature required to simultaneously achieve these parameters, assuming a value for $q_{\min }, Z_{\mathrm{eff}}$ and the density and temperature profile shapes. Once the average density and temperature are known, all of the typical parameters used to characterize the plasma performance and operational space, such as $H_{98 y 2}, f_{\mathrm{DL}}, \beta_{\mathrm{N}}$, and proximity to the L-H power threshold scaling, can be derived. In the case discussed here, the plasma current $I$ is left as a free parameter. To make figure 14, the goals of 3000 s operation at $Q=5$ with $50 \mathrm{MW}$ of auxiliary heating power were specified. This method indicates that simultaneously operating below the density limit and keeping the loss power higher than the L-H threshold power at the operational point requires $I>10.5 \mathrm{MA}$ (shaded region of the plot). The values of $\beta_{\mathrm{N}}$ for the self-consistent solution are in the range of 2.0-2.3 (not shown), and the confinement implied is a modest improvement over that given by the H-mode scaling $\left(H_{98 \mathrm{y} 2}=1.1-1.3\right)$, indicating that advanced inductive performance may not be required to meet the minimum goals of hybrid operation in ITER, but may lead to higher neutron fluence. The plot shows the choices confronting the scenario designer to meet the specified goals. Lower current operation is attractive to reduce the impact of a disruption on the tokamak, but requires better confinement closer to both the empirical density limit and the L-H power threshold. Higher current operation moves away from these limits, but challenges divertor operation by not running the maximum density allowed under the empirical limit. The insight 
provided by this analysis into the influence of simultaneous operational constraints is quite helpful in designing operational scenarios to meet specific goals. This is the key result from this modeling activity, in contrast to focusing on the precise value of the performance prediction. The analysis presented here also indicates that the plasma performance required for advanced inductive operation with $P_{\text {fus }}=250 \mathrm{MW}$ approaching 1 hour in duration in ITER is within the existence domain of experimental results shown previously in terms of dimensionless parameter metrics.

It has been suggested that the shape of the current profile could be the origin of both the improved confinement (and perhaps the variation in $H_{98 \mathrm{y} 2}$ seen in the dataset) and the improved stability to $n=1$ tearing modes $[16,38,39]$. The best measurements of the current profile from all four tokamaks indicate the $q$ profile is flat in the core with $\min (q)$ very close to 1 . Modeling on AUG [35] and DIII-D [40] indicated the stationary current profile measured after many $\tau_{\mathrm{R}}$ is not consistent with the profile found by modeling the current evolution with neoclassical Ohm's law and the standard models for the noninductive external current drive sources using the measured density and temperature profiles. The modeled current density profile under those assumptions has $\min (q)$ significantly below 1 . The measured current profile is less peaked, consistent with the small or non-existent sawteeth that are related to a $q=1$ surface in the plasma. Modeling of JT-60U plasmas using a constant $Z_{\text {eff }}$ profile also predicts $\min (q)$ significantly below 1 after many $\tau_{R}$, but estimates of $Z_{\text {eff }}$ that include a centrally peaked tungsten density (coming from the ferritic inserts used in JT-60U to reduce the toroidal magnetic field ripple) would allow the possibility for $\min (q) \sim 1$ in stationary conditions. In contrast, modeling of JET plasmas [9] appears consistent with expectations of current evolution modeled using the standard theory. Enhancements in the JET NBI capability now coming available should allow a more definitive 
statement on this discrepancy by extending the plasma duration relative to $\tau_{\mathrm{R}}$ beyond what was possible at the time the ITPA database was collected. It is plausible to expect enhanced confinement to accompany a flatter $q$ profile, since lower transport from drift waves is predicted [41]. That the current profile should have an effect on tearing mode stability is also plausible, but the theory of tearing stability in high- $\beta$ toroidal plasmas is not validated sufficiently to provide an optimization strategy for the current profile. Two experiments on DIII-D, alternately suppressing or enhancing the $n=2$ tearing mode amplitude with electron cyclotron current drive (ECCD) [12] and measuring the impact of ELMs on the central current profile through the $n=2$ tearing mode [42] indicate that this mode plays a key role in the current profile evolution beyond that expected from a simple modification of the resistivity profile through local energy transport. Fishbones are seen to play a similar role in AUG plasmas [43]. Therefore, MHD modes play both a desirable role (avoiding $q=1$ ) and an undesirable role (reducing confinement, limiting pressure) in these plasmas.

\section{Discussion and conclusions}

Many open questions regarding the physics basis of the present-day experiments and the estimation of performance in ITER remain. One of the most frequently asked questions is whether these advanced inductive plasmas constitute a new operating regime. The evolution of the pressure and current profile, connected through the resistive equilibrium, to a state favorable to both energy confinement and tearing mode stability appears to be a distinctive feature of these plasmas. While sawteeth prevent conventional $\mathrm{H}$-mode plasmas from reaching resistive equilibrium, the evolution between sawteeth appears to be described well by standard theory models. Advanced inductive plasmas often stop evolving before sawteeth appear, inhibited by a mechanism connected to the other MHD behavior in the plasma. This difference in the current 
profile evolution may be connected with the favorable energy transport. Just as the H-mode pedestal allows the plasma to access a new region of what seems to be a continuum of energy transport behavior, this modification of the stationary current profile may lead to previously unseen energy transport behavior by accessing current profiles with improved stability to the $n=1$ tearing mode, albeit without an analogous threshold behavior to the H-mode transition. While some outstanding issues are in common with those of conventional H-mode operation (pedestal behavior, radiative divertor operation, ELM mitigation), the IOS has tried to focus on those unique to these plasmas. For example, prescriptions for reaching advanced inductive performance exist for each of the tokamaks represented here; but access conditions that are necessary and sufficient to reach this regime in ITER have yet to be defined. Initial experiments and the database analysis shown here indicate transport behavior different from that seen in conventional H-mode. The standard H-mode thermal confinement scaling does not organize the data well and unexplained variations with key dimensionless parameters (normalized gyroradius and collision frequency) that vary significantly from present-day experiments to ITER are seen. Resolving these issues by joint experiments and modeling activities remains a high priority task for the IOS group in the near future.

Despite uncertainties in the details, some clear conclusions can be drawn from the present information. The data collected by the IOS group indicates that these advanced inductive scenarios are a robust mode of operation in divertor tokamaks. This conclusion rests on the fact that plasmas meeting the criteria for being designated as advanced inductive have been obtained across the range of normalized density and current of interest to fusion energy experiments and power plants. The distinguishing characteristics of this mode of operation are stationary operation on the time scale of a fully-relaxed current profile at higher $\beta_{\mathrm{N}}$ than obtained in 
conventional $\mathrm{H}$ mode operation at low $q_{95}$, while maintaining good confinement quality. The projections discussed here indicate this mode of operation has promise to fulfill the fluence goals of the ITER project, but may also provide a lower-risk alternative approach to achieving the primary physics objective of $Q=10$ operation at $500 \mathrm{MW}$ fusion power for $400 \mathrm{~s}$.

\section{Acknowledgment}

This work was supported in part by the US Department of Energy under DE-FC0204ER54698, DE-AC02-09CH11466, DE-AC05-00OR22725, and DE-AC05-06ER23100 and by EURATOM within the framework of the European Fusion Development Agreement. The views and opinions expressed herein do not necessarily reflect those of the European Commission. The authors gratefully acknowledge the contributions of the ASDEX-Upgrade team, the DIII-D team, the JET EFDA Contributors, and the JT-60U team without whom this work would not be possible. The International Tokamak Physics Activity now operates under the ITER International Organization. Views and opinions expressed herein do not necessarily reflect those of the ITER Organization.

\section{Appendix. Database Description}

Data from four divertor tokamaks (AUG, DIII-D, JET, JT-60U) were compiled through the auspices of the ITPA in order to compare the performance and operational regimes of advanced inductive scenarios. No selection criteria for inclusion were imposed; however, the goal to study stationary plasmas with good confinement at $\beta_{\mathrm{N}}$ significantly above that assumed for the ITER baseline scenario $\left(\beta_{\mathrm{N}}=1.8\right)$ was clearly stated to the data providers. The data provided are considered representative of the advanced inductive performance of each tokamak, but not a 
comprehensive description of the performanace achieved in each tokamak. This appendix is intended to provide a description of the data supplied; analysis of the database is in the main text.

The data are characterized here by the range and distribution of values of the plasma parameters typically used for energy confinement scaling relations. Table A1 lists these parameters and their ranges for each tokamak for plasmas meeting the criteria $\beta_{\mathrm{N}} \geq 2.4$ for duration at least $5 \tau_{\mathrm{E}}$. Here, $R$ is the major radius of the geometric center of the plasma boundary, $a$ is the minor radius of the plasma boundary, $\kappa$ is the elongation at the separatrix, $B$ is the vacuum toroidal magnetic field at $R, I$ is the plasma current, $n$ is the line-averaged electron density, and $P$ is the input power. From Table A1, it is clear that $R$ and $a$ do not vary appreciably within the data for each tokamak. The value of $\kappa$ does vary somewhat, but the distribution is peaked around 1.8 for AUG, DIII-D, and JET, while the data from JT-60U peaks at a slightly lower value of 1.5 . For parameters with significant variation in the subset of data from each tokamak ( $I, B, n, P)$, figure A1 illustrates the distribution of values. The values of $B$ and $I$ tend to cluster around discrete values, since these are set prior to the plasma initiation and are well regulated. The value of density is more widely distributed, due both to lower capability for tight regulation but also through experiments designed to vary it. The value of power also varies more significantly; some of this is due to operation under feedback control to yield a specified stored energy. 


\section{References}

[1] Shimada M. et al 2007 Nucl. Fusion 47 S1

[2] Gormezano C. et al 2007 Nucl. Fusion 47 S285

[3] Troyon F. et al 1984 Plasma Phys. Control. Fusion 26209

[4] ITER Physics Basis 1999 Nucl. Fusion 392175

[5] Yushmanov P.N. et al 1990 Nucl. Fusion 301999

[6] Greenwald M. 2002 Plasma Phys. Control. Fusion 44 R27

[7] Sips A.C.C. et al 2002 Plasma Phys. Control. Fusion 44 B69

[8] Luce T.C. et al 2004 Phys. Plasmas 112627

[9] Joffrin E. et al 2005 Nucl. Fusion 45626

[10] Oyama N. et al 2009 Nucl. Fusion 49065026

[11] Gruber O. et al 2009 Nucl. Fusion 49115014

[12] Wade M.R. et al 2005 Nucl. Fusion 45407

[13] Staebler G.M. et al 2005 Nucl. Fusion 45617

[14] Maget P. et al 2010 Nucl. Fusion 50045004

[15] Kamada Y. et al 1999 Nucl. Fusion 391845

[16] Luce T.C. et al 2003 Nucl. Fusion 43321

[17] Luce T.C. et al 2008 Plasma Phys. Control. Fusion 50043001

[18] Joffrin E. 2007 Plasma Phys. Control. Fusion 49 B629

[19] Petty C.C. et al 2003 Fusion Sci. Technol. 431

[20] Politzer P.A. et al 2010 Proc. 23rd IAEA Fusion Energy Conference, Daejeon, paper EXC/P2-06 
[21] Petty C.C. 2008 Proc. 22 $2^{\text {nd }}$ IAEA Fusion Energy Conference, Geneva, paper EX1-4rb, http://www-naweb.iaea.org/napc/physics/FEC/FEC2008/papers/ex 1-4rb.pdf

[22] Sips A.C.C. et al 2007 Nucl. Fusion 471485

[23] Politzer P.A. et al 2008 Nucl. Fusion 48075001

[24] Maggi C. et al 2007 Nucl. Fusion 47535

[25] Hudson B. et al 2010 Nucl. Fusion 50045006

[26] Schweinzer J. et al 2011 Nucl. Fusion 51113003

[27] Corre Y. et al 2008 Plasma Phys. Control. Fusion 50115012

[28] Petrie T.W. et al 2007 J. Nucl. Mater. 363-365 416

[29] Kessel C.E. et al 2007 Nucl. Fusion 471274

[30] Pereverzev G. et al 2002 IPP-Report IPP 5/98

[31] Artaud J.F. et al 2010 Nucl. Fusion 50043001

[32] Hayashi N. and JT-60 Team 2010 Phys. Plasmas 17056112

[33] Kessel C.E. et al 2006 Phys. Plasmas 13056108

[34] Imbeaux F. et al 2005 Plasma Phys. Control. Fusion 47 B179

[35] Na Yong-Su et al 2006 Nucl. Fusion 46232

[36] Citrin J. et al 2010 Nucl. Fusion 50115007

[37] Polevoi A.R. et al 2010 Proc. of $37^{\text {th }}$ EPS Conf. on Plasma Physics, Dublin, Ireland (EPS) P2.187.

[38] Stober J. et al 2007 Nucl. Fusion 47728

[39] Joffrin E. et al 2008 Proc. of 22nd IAEA Fusion Energy Conf., Geneva, paper EX1-4Ra, http://www-naweb.iaea.org/napc/physics/FEC/FEC2008/papers/ex_1-4ra.pdf

[40] Casper T.A. et al 2007 Nucl. Fusion 47825 
[41] Waltz R.E. et al 1995 Phys. Plasmas 22408

[42] Petty C.C. et al 2009 Phys. Rev. Lett. 102045005

[43] Guenter S. et al 1999 Nucl. Fusion 391535 
Table 1. Ranges of variables in the two $v^{\dagger}$ scans found in the DIII-D advanced inductive data set. The first three should be constant, while $v^{\dagger} \propto B^{-4}$ in a proper $v^{\dagger}$ scan. The range in $B$ implies a scan in $v^{\dagger}$ of 2.4 for Group 1 and 2.1 for Group 2.

\begin{tabular}{|l|c|c|c|c|}
\hline \hline & $\begin{array}{c}\boldsymbol{n} \\
\left(\mathbf{1 0} / \mathbf{m}^{\mathbf{3}}\right)\end{array}$ & $\begin{array}{c}\mathbf{I} / \boldsymbol{B} \\
(\mathbf{M A} / \mathbf{T})\end{array}$ & $\begin{array}{c}\boldsymbol{\beta} \\
(\boldsymbol{\%})\end{array}$ & $\begin{array}{c}\mathbf{B} \\
(\mathbf{T})\end{array}$ \\
\hline Group 1 & $6.7-7.0$ & $0.68-0.70$ & $3.0-3.1$ & $1.72-2.15$ \\
Group 2 & $5.5-6.0$ & $0.64-0.70$ & $2.9-3.0$ & $1.78-2.15$ \\
\hline \hline
\end{tabular}


Table 2. Correlations among the fit variables used in the regression analysis for confinement scaling.

\begin{tabular}{|l|l|l|l|l|l|}
\hline \hline & $\operatorname{In}\left(\boldsymbol{I}_{\mathbf{p}}\right)$ & \multicolumn{1}{|c|}{$\operatorname{In}\left(\boldsymbol{B}_{\mathrm{T}}\right)$} & \multicolumn{1}{|c|}{$\operatorname{In}\left(\boldsymbol{P}_{\mathrm{in}}\right)$} & \multicolumn{1}{|c|}{$\operatorname{In}(\boldsymbol{R})$} & \multicolumn{1}{|c|}{$\operatorname{In}(\boldsymbol{a})$} \\
\hline $\operatorname{In}\left(I_{\mathrm{p}}\right)$ & 1.0000 & -0.0285 & 0.4012 & 0.4927 & 0.6722 \\
$\operatorname{In}\left(B_{\mathrm{T}}\right)$ & -0.0285 & 1.0000 & 0.4694 & 0.0697 & -0.1448 \\
$\operatorname{In}\left(P_{\mathrm{in}}\right)$ & 0.4012 & 0.4694 & 1.000 & 0.6960 & 0.5545 \\
$\operatorname{In}(R)$ & 0.4927 & 0.0697 & 0.6960 & 1.000 & 0.9294 \\
$\operatorname{In}(a)$ & 0.6722 & -0.1448 & 0.5545 & 0.9294 & 1.0000 \\
\hline \hline
\end{tabular}


Table A1. Ranges of values for plasma parameters in the database. The numbers in parentheses following the tokamak names are the number of entries in the database satisfying the selection criteria defined in the text.

\begin{tabular}{|l|l|l|l|l|l|l|l|l|}
\hline \hline Parameter & \multicolumn{2}{|c|}{ AUG (127) } & \multicolumn{2}{c|}{ DIII-D (279) } & \multicolumn{2}{c|}{ JET (162) } & \multicolumn{2}{c|}{ JT-60U (21) } \\
\hline & Min & Max & Min & Max & Min & Max & Min & Max \\
\hline$R(\mathrm{~m})$ & 1.59 & 1.64 & 1.66 & 1.73 & 2.89 & 2.97 & 3.22 & 3.41 \\
\hline$a(\mathrm{~m})$ & 0.47 & 0.52 & 0.54 & 0.62 & 0.88 & 0.95 & 0.78 & 0.93 \\
\hline $\mathrm{K}$ & 1.59 & 1.91 & 1.70 & 1.94 & 1.58 & 1.83 & 1.39 & 1.54 \\
\hline$B(\mathrm{~T})$ & 1.39 & 2.79 & 1.01 & 2.15 & 1.12 & 2.27 & 1.54 & 4.06 \\
\hline$I(\mathrm{MA})$ & 0.60 & 1.21 & 0.68 & 1.50 & 0.90 & 2.0 & 0.85 & 1.80 \\
\hline$n\left(10^{19} \mathrm{~m}^{-3}\right)$ & 4.4 & 11.9 & 2.5 & 7.4 & 2.2 & 7.2 & 1.5 & 4.0 \\
\hline$P(\mathrm{MW})$ & 4.7 & 18.7 & 3.0 & 10.8 & 7.4 & 22.1 & 6.6 & 22.3 \\
\hline \hline
\end{tabular}




\section{Figure Captions}

FIG. 1. Longest duration $\left(>15 \tau_{\mathrm{R}}\right)$ advanced inductive plasma (from the JT-60U tokamak). [Reprinted courtesy of Institute of Publishing, N. Oyama, et al., Nucl. Fusion 49 (2009) 065026 by permission].

FIG. 2. Distribution of advanced inductive plasmas in the operating space defined by normalized plasma current and density.

FIG. 3. Distribution of advanced inductive plasmas in the operating space defined by normalized pressure and internal inductance.

FIG. 4. Achieved $\beta_{\mathrm{N}}$ in advanced inductive plasmas vs proxies for dimensionless (a) gyroradius, (b) collision frequency, and (c) plasma current as defined in the text.

FIG. 5. $G$ vs proxies for dimensionless (a) gyroradius, (b) thermal pressure, (c) collision frequency, and (d) plasma current as defined in the text. The horizontal cyan bar in each box indicates the location of $G=0.42$, which is the value for $Q=10$ in ITER. The shaded regions in $(a-c)$ show the range of values for the proxy in ITER. The vertical bars in (d) show equivalent values for the different plasma currents in ITER as indicated.

FIG. 6. $H_{98 y 2}$ vs proxies for dimensionless (a) gyroradius, (b) thermal pressure, (c) collision frequency, and (d) plasma current as defined in the text. The horizontal cyan line in each box indicates the location of $H_{98 \mathrm{y} 2}=1$. The shaded regions in $(\mathrm{a}-\mathrm{c})$ show the range of values for each proxy in ITER. The vertical bars in (d) show equivalent values for the different plasma currents in ITER as indicated. 
FIG. 7. Comparison of the dataset values of the dimensionless gyroradius proxy vs the proxy for the dimensionless collision frequency.

FIG. 8. $H_{\mathrm{DS} 03}$ vs proxies for dimensionless (a) gyroradius, (b) thermal pressure, (c) collision frequency, and (d) plasma current as defined in the text. The horizontal cyan bar in each box indicates the location of $H_{\mathrm{DS} 03}=1$. The shaded regions in $(\mathrm{a}-\mathrm{c})$ show the range of values for the proxy in ITER. The vertical bars in (d) show equivalent values for the different plasma currents in ITER as indicated.

FIG. 9. Scaling of $v^{\dagger}$ in DIII-D advanced inductive plasmas as shown by plotting $\ln \left(B \tau_{\text {th }}\right)$ vs $\ln \left(C v^{\dagger}\right)$. The diamonds are the five cases from Group 1 in Table 1 and the squares are the five cases from Group 2. The best linear fit (points equally weighted) to each group is shown by the straight lines. Group 1 has $B \tau_{\text {th }} \propto\left(v^{\dagger}\right)^{-0.44}$; Group 2 has $B \tau_{\text {th }} \propto\left(v^{\dagger}\right)^{-0.56}$.

Fig. 10. $H_{98 \mathrm{y} 2}$ vs the ratio of the ion to electron temperature for the (a) AUG, (b) DIII-D, and (c) JET tokamaks.

Fig. 11. $H_{98 \mathrm{y} 2}$ vs dimensionless proxy for the Mach number for the (a) AUG, (b) DIII-D, (c) JET, and (d) JT-60U tokamaks.

Fig. 12. (a) Measured thermal confinement time vs the three-parameter fit with $R$ as the length parameter. (b) Measured thermal confinement time vs the three-parameter fit with $a$ as the length parameter. 
Fig. 13. Profiles of predicted (a) electron and ion temperature, (b) current density, and (c) $q$ for the modeling benchmark tests described in the text.

Fig. 14. ITER operational space diagram for advanced inductive operation at the nominal vacuum toroidal magnetic field of $B=5.3 \mathrm{~T}$. The black curve is the ratio of the density to the density limit, the green curve is the ratio of the loss power to the predicted L-H threshold power, and the red curve is the confinement quality measured by the H-mode scaling.

Fig. A1. Histograms of the plasma current $I$ (far left column), magnetic field $B$ (middle left column), line-averaged density $n$ (middle right column), and input power $P$ (far right column from AUG (top row), DIII-D (upper middle row), JET (lower middle row), and JT-60U (bottom row) for plasmas that satisfy the selection criteria defined in the text. 


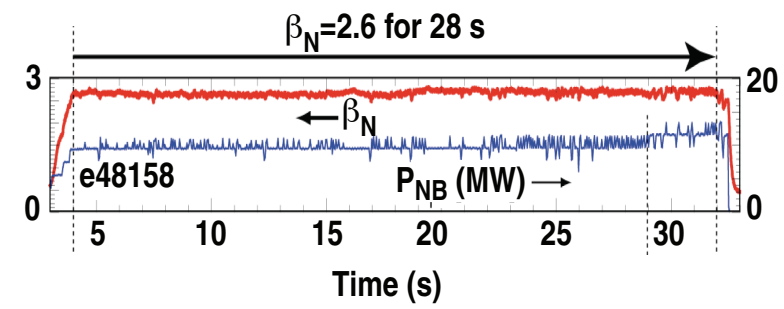

102-12 f1 jy 


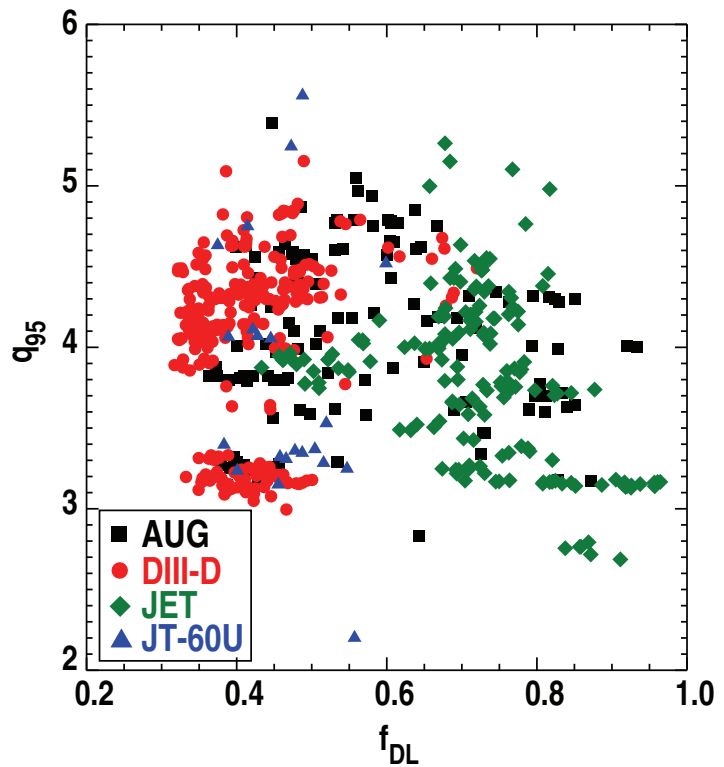

102-12 f2 jy 


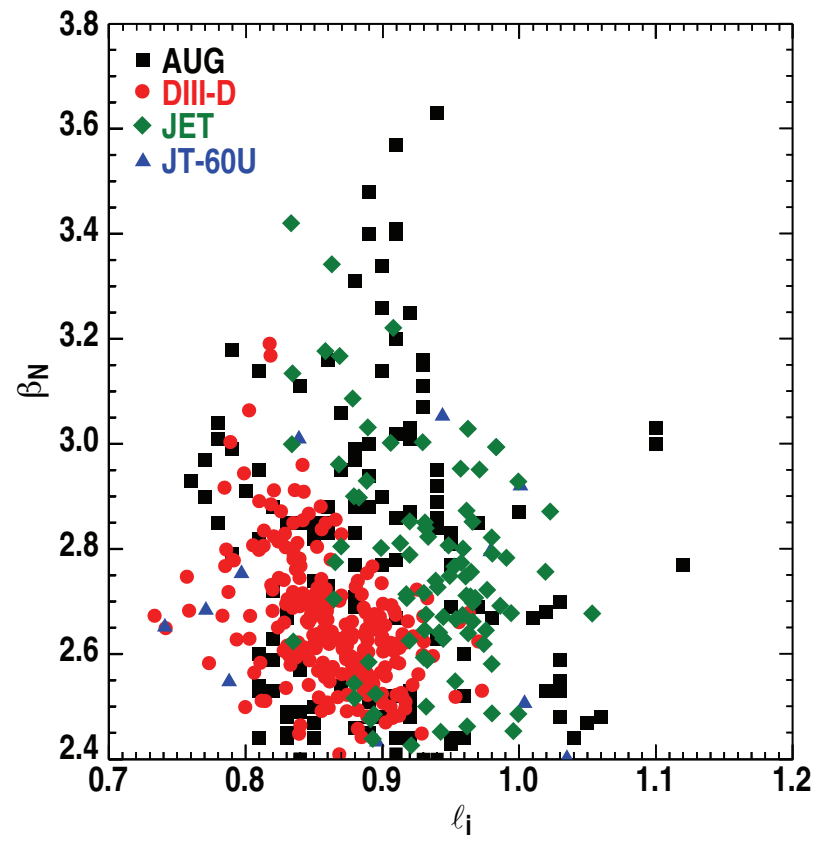

102-12 f3 jy 

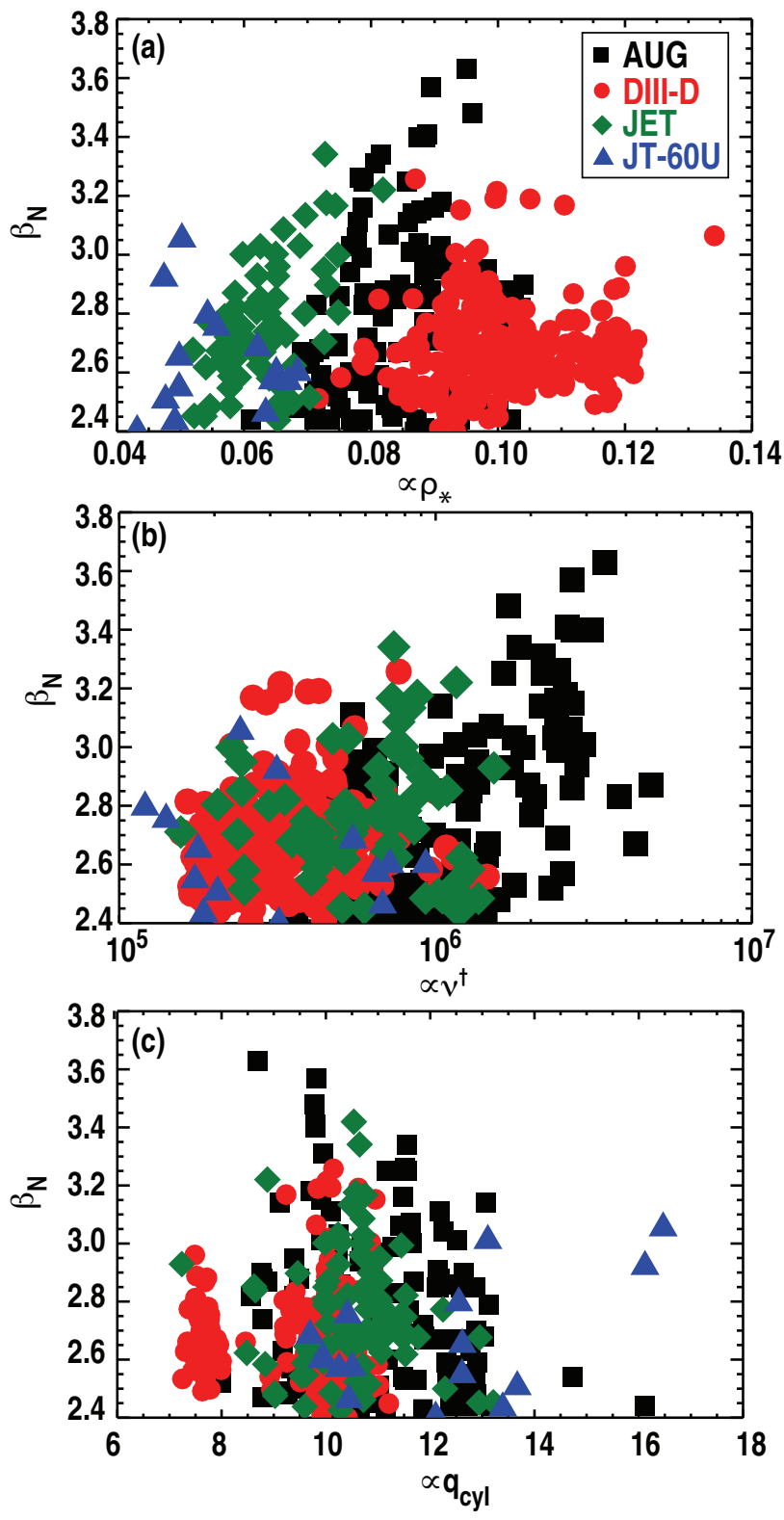

102-12 f4 jy 

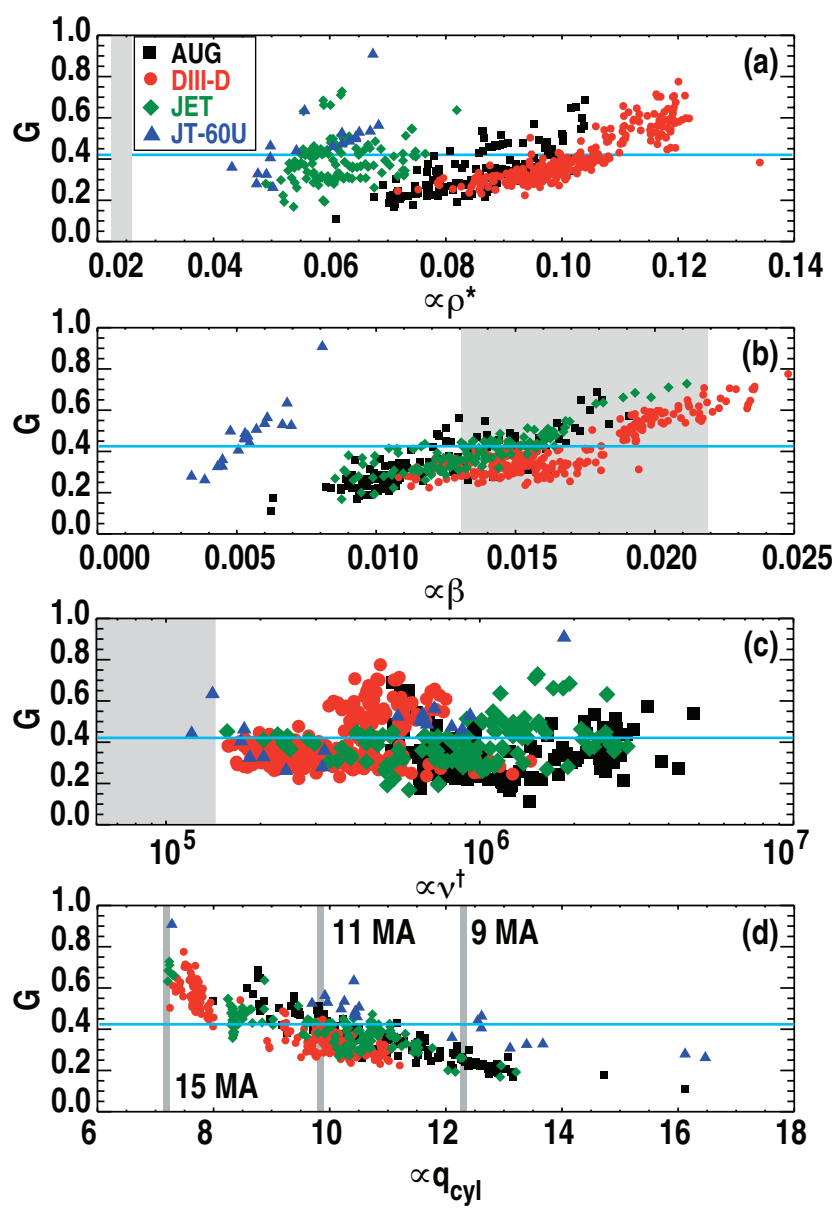

102-12 f5 jy 

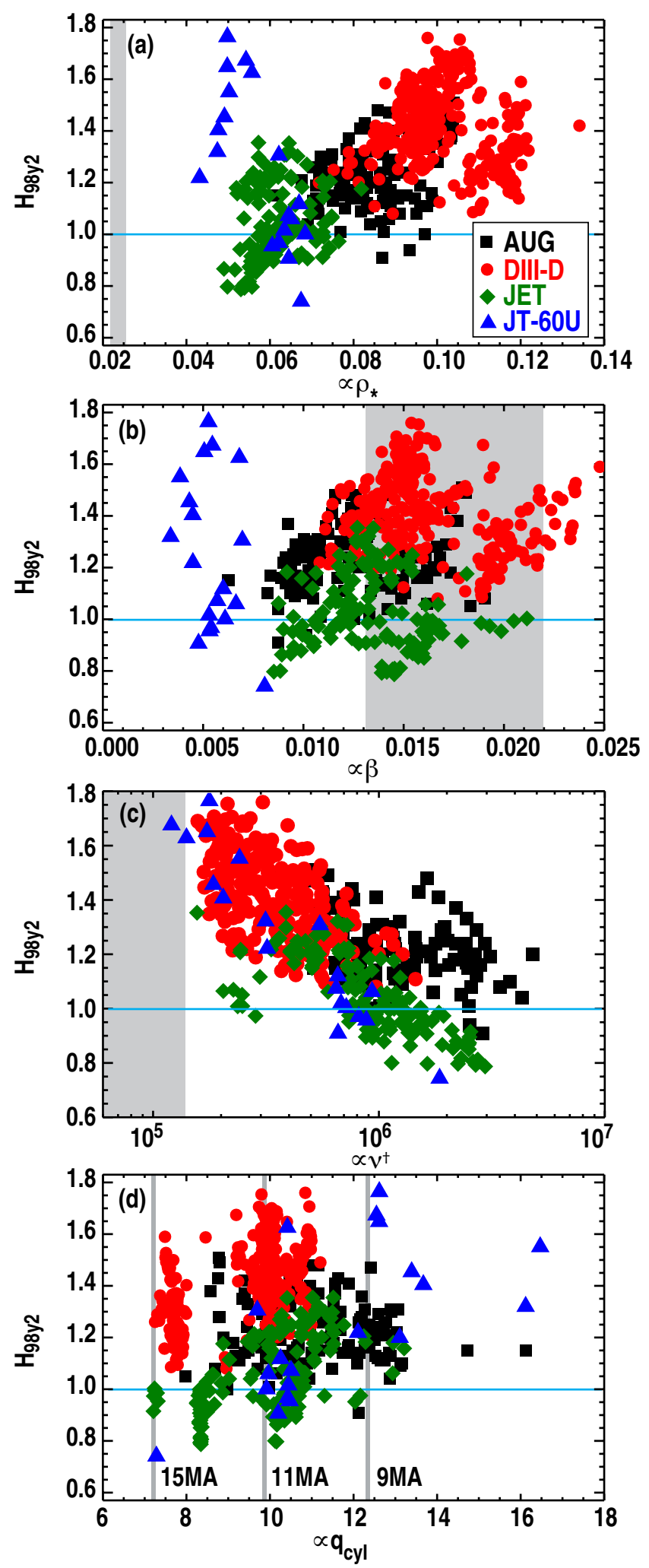

102-12 f6 jy 


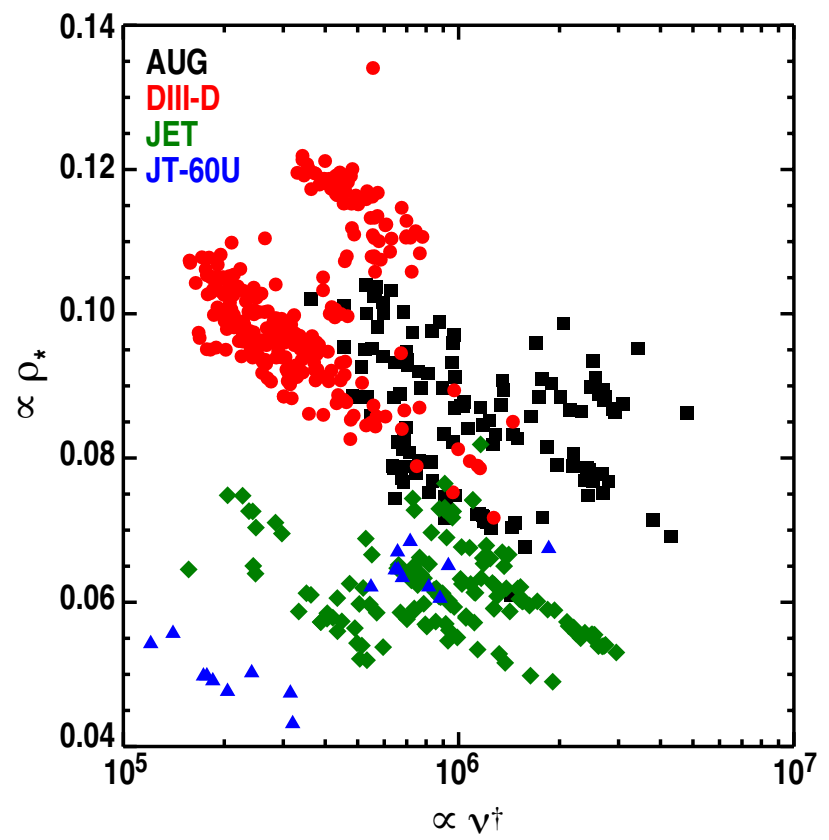

102-12 f7 jy 

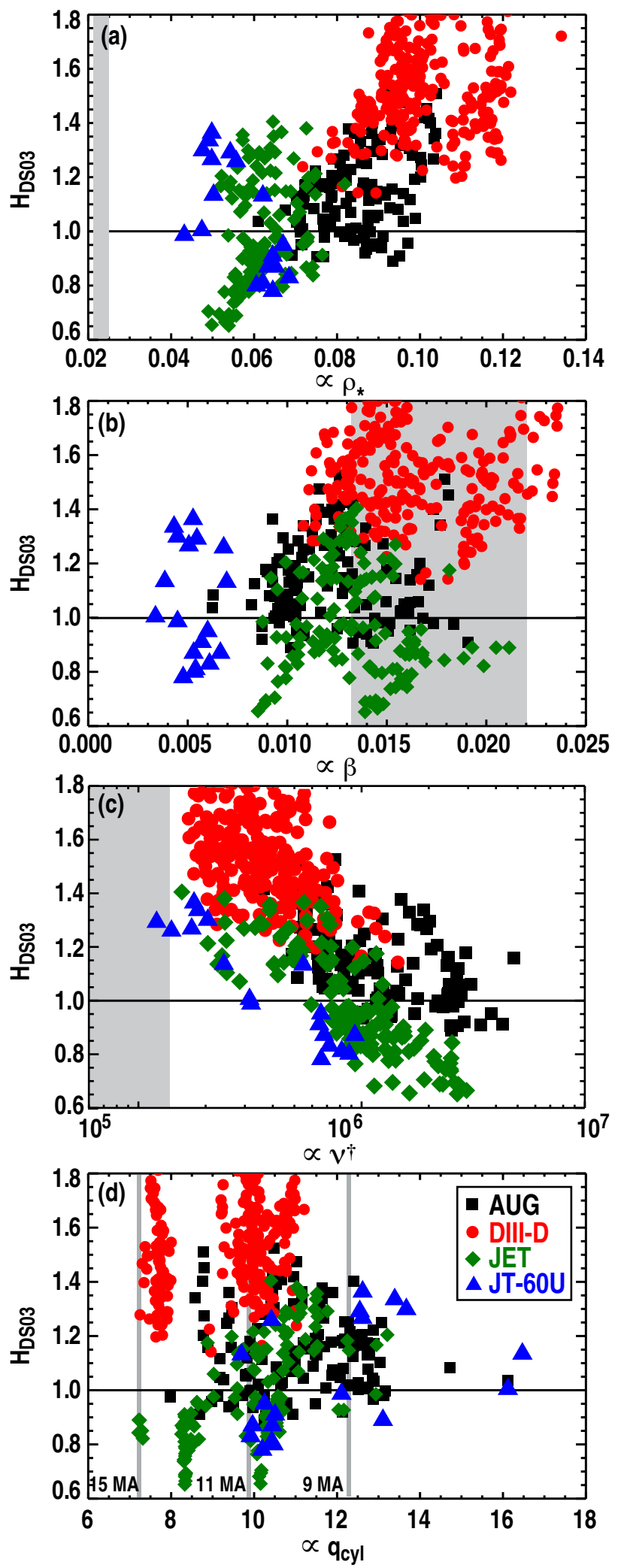

102-12 f8 jy 


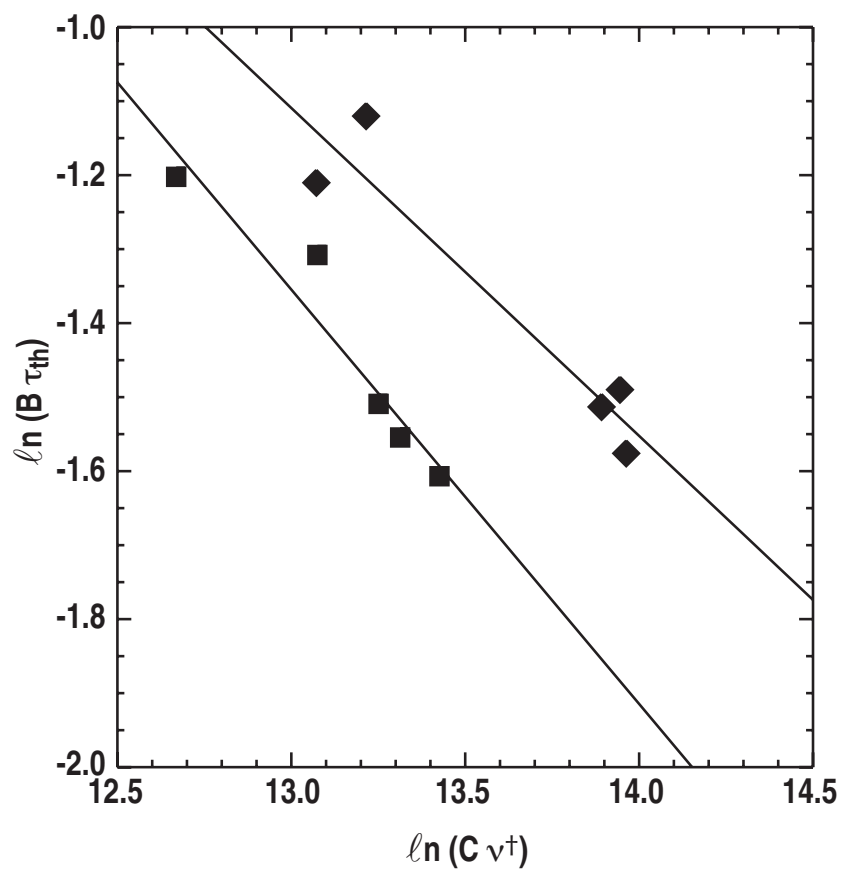

102-13 f9 jy 


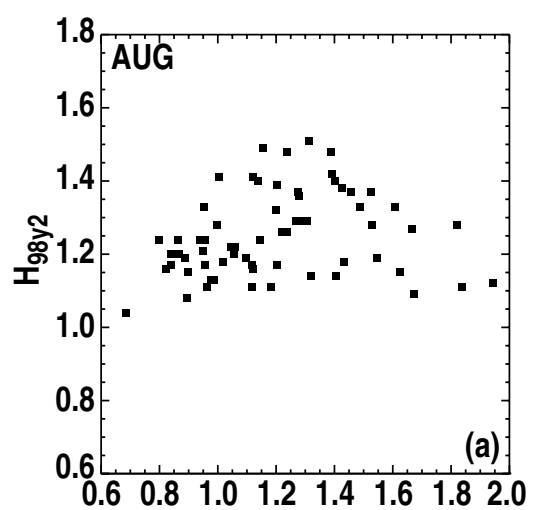

$\mathrm{T} \mathrm{i} / \mathrm{Te}$
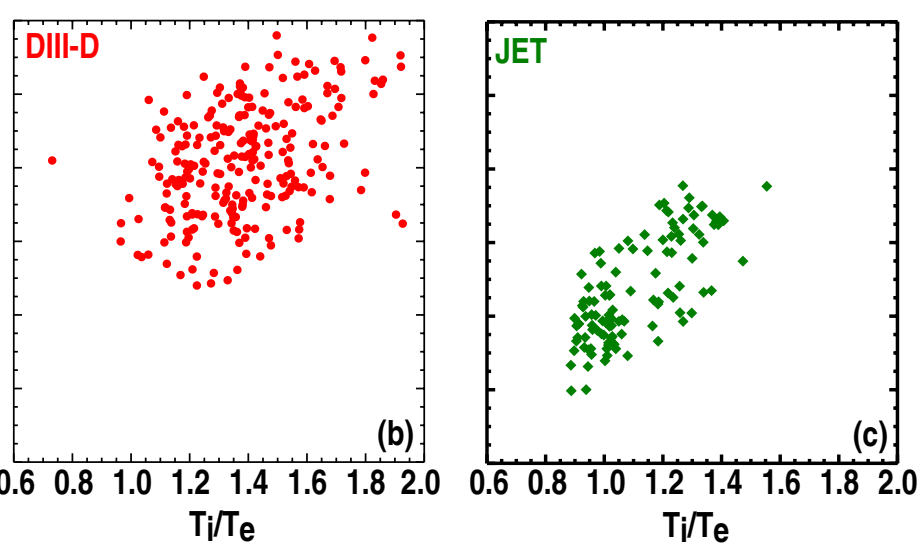

102-12 f10 jy 

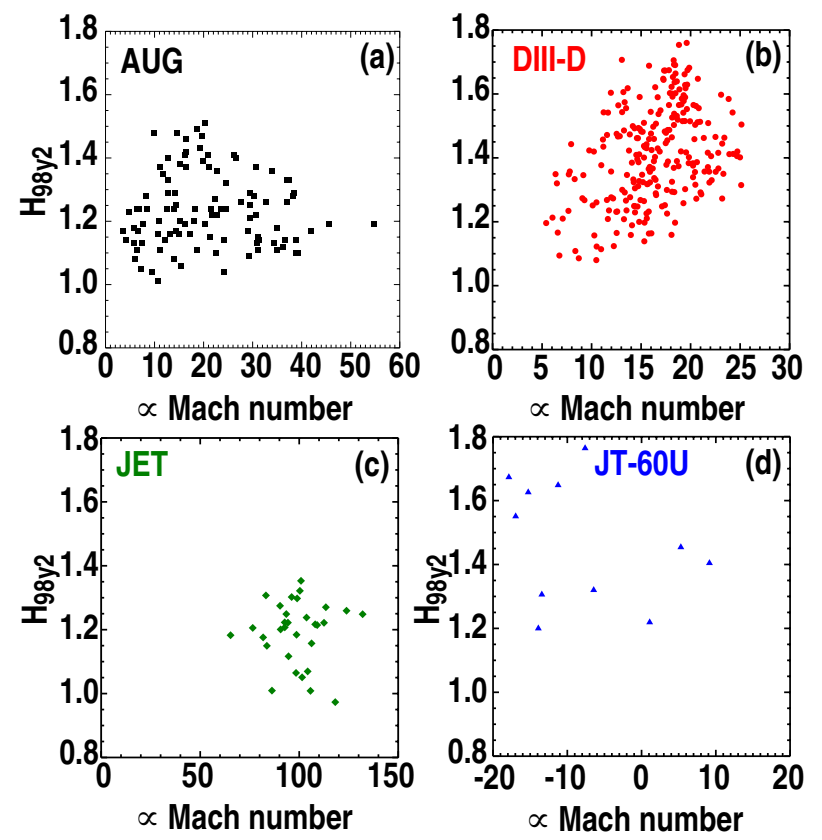

102-12 f11 jy 

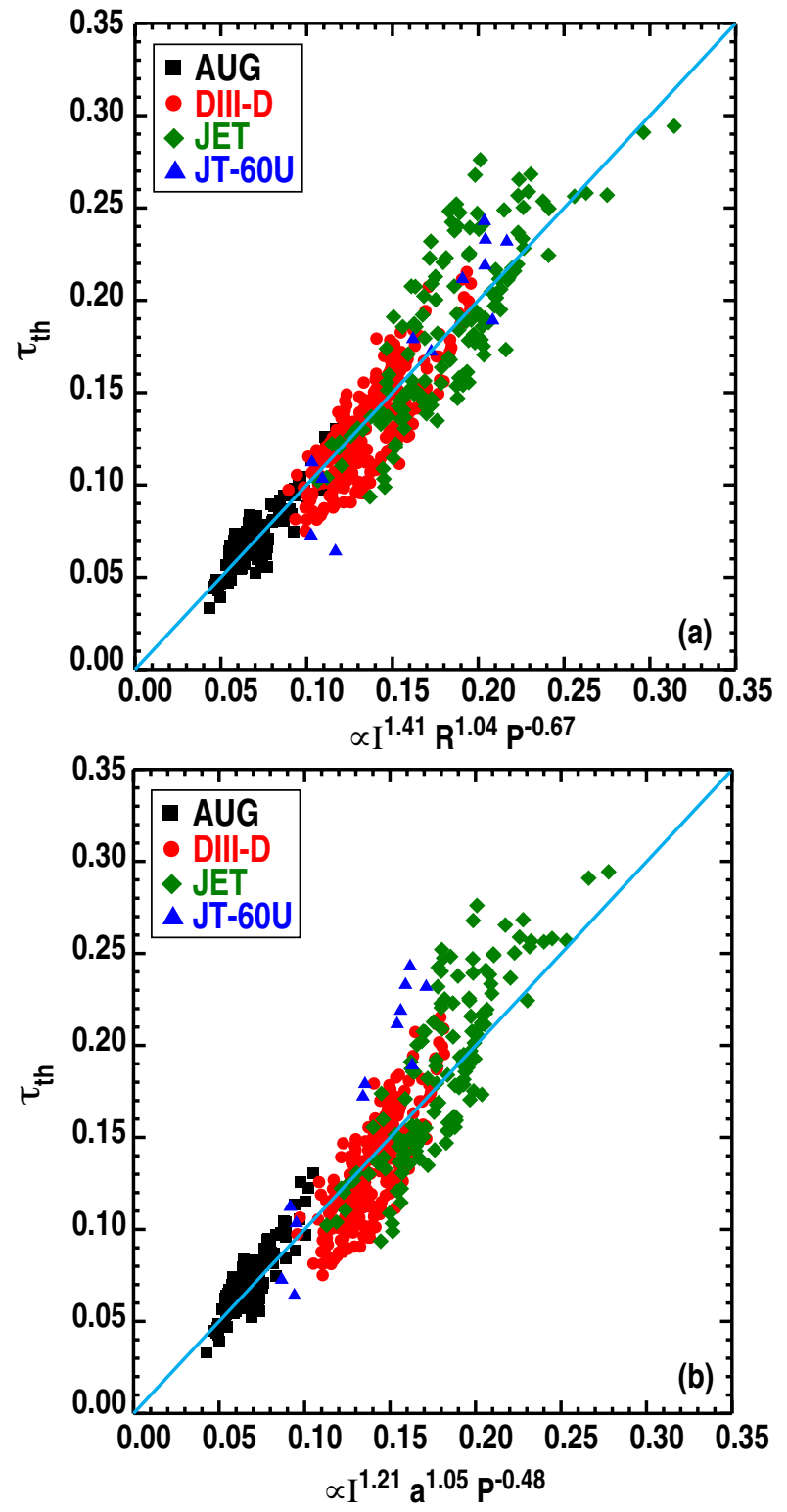

102-12 f12 jy 

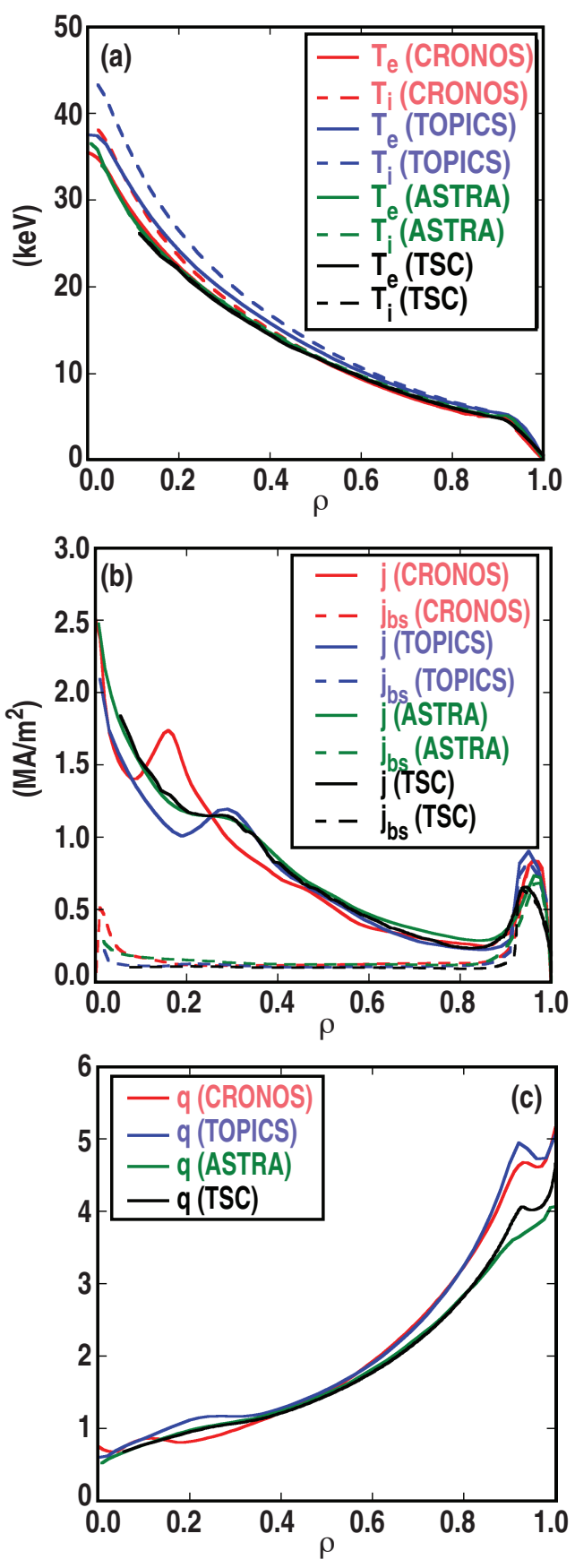

102-13 f13 jy 


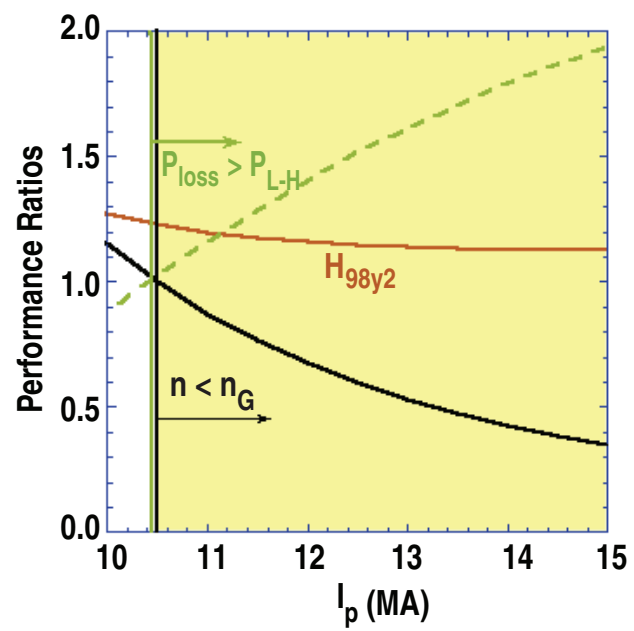

102-12 f14 jy 

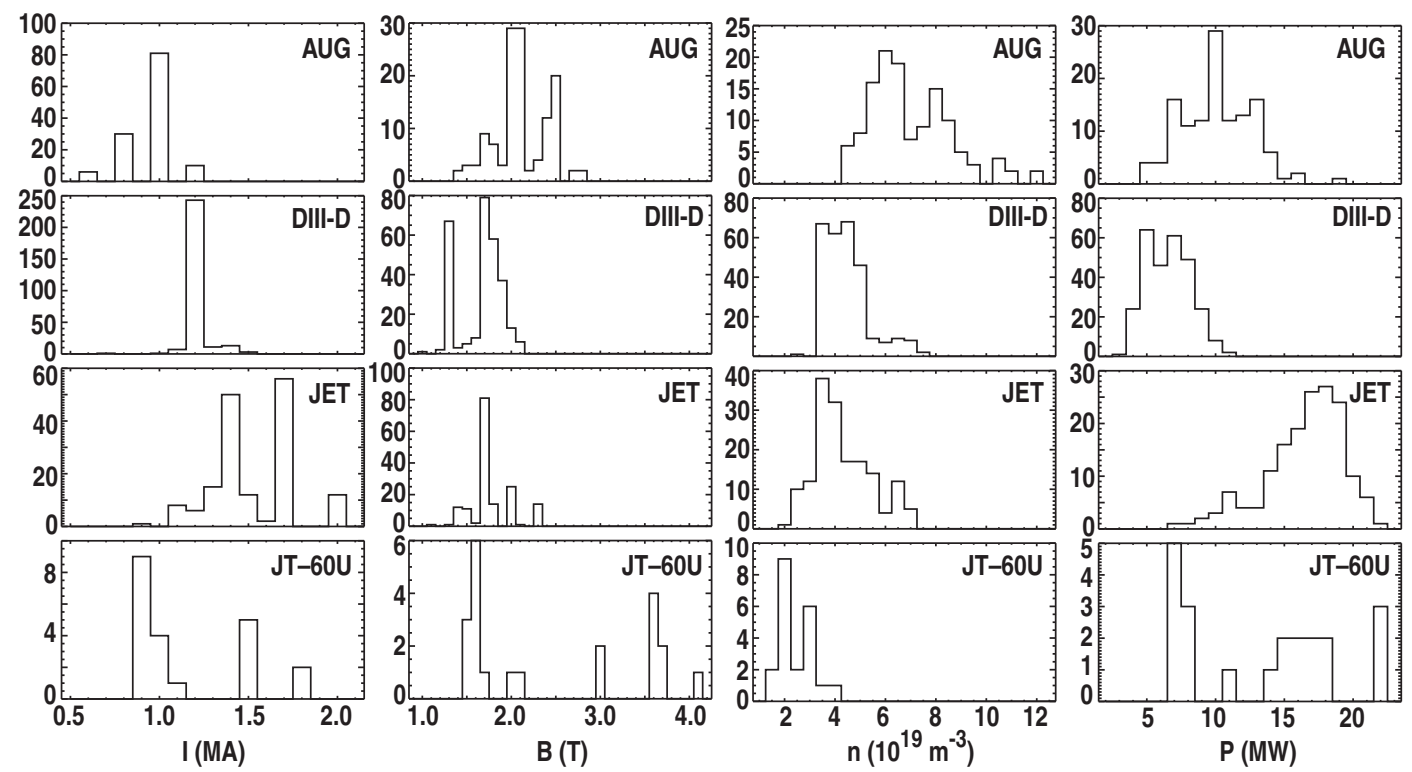

102-12 A1 jy 